DESY $97-110$

ISSN 0418-9833

$\mathrm{MPI} / \mathrm{PhT} / 97-001$

hep-ph/9706369

\title{
Charmonium Production via Fragmentation at DESY HERA
}

\author{
Bernd A. KNiehl ${ }^{1}$ And Gustav Kramer ${ }^{2}$ \\ ${ }^{1}$ Max-Planck-Institut für Physik (Werner-Heisenberg-Institut), \\ Föhringer Ring 6, 80805 Munich, Germany \\ 2 II. Institut für Theoretische Physik, Universität Hamburg, \\ Luruper Chaussee 149, 22761 Hamburg, Germany
}

\begin{abstract}
The cross section for the photoproduction of large- $p_{T} J / \psi$ mesons at HERA is calculated at next-to-leading order, adopting a perturbative approach to describe the fragmentation of charm quarks and gluons into $J / \psi$ mesons. We treat the charm quark according to the massless factorization scheme, where it is assumed to be one of the active flavours inside the proton and the resolved photon. We present inclusive distributions in transverse momentum and rapidity, including the contributions due to direct and resolved photons. The importance of the colouroctet components of the $J / \psi$ wave function, which contribute to the fragmentation process, is emphasized. In addition to prompt $J / \psi$ production, we consider also the production of $\chi_{c J}$ states followed by radiative decays to $J / \psi$ mesons, both in the colour-singlet and colour-octet channels.
\end{abstract}

PACS numbers: 13.60.-r, 13.85.Ni, 13.87.Fh, 14.40.Lb

*Supported by Bundesministerium für Forschung und Technologie, Bonn, Germany, under Contract $057 \mathrm{HH}$ 92P (5), and by EU Program Human Capital and Mobility through Network Physics at High Energy Colliders under Contract CHRX-CT93-0357 (DG12 COMA). 


\section{Introduction}

The production of heavy quarkonia in high-energy collision has conventionally been described within the colour-singlet model (CSM) [1]. In this model, a non-relativistic approximation is used to describe the binding of the heavy-quark pair, produced via partonfusion processes, into a quarkonium state. The heavy-quark pair is projected onto a physical quarkonium state using a colour-singlet projection and an appropriate spin projection. Experimentally, $J / \psi$ production is measured most easily through the detection of the leptonic decays. Then, the inclusive $J / \psi$ yield consists of the prompt $J / \psi$ production and the production of the $c \bar{c}^{3} P_{J}$ states $\chi_{c J}(J=0,1,2)$, which decay electromagnetically into the lower-lying $J / \psi$ state. The latter must also be taken into account if one wishes to compare theoretical predictions with experiments where $\chi_{c J}$ production could not be measured separately. The CSM has been successfully applied [2] to explain large- $p_{T} J / \psi$ production at the relatively low energies $(\sqrt{s}=63 \mathrm{GeV})$ of the CERN ISR experiments. After the advent of the CERN $S p \bar{p} S$ collider with $\sqrt{s}=630 \mathrm{GeV}$, it was found [3] that also the production of bottom quarks and their subsequent weak decay into $J / \psi$ mesons is an important source of $J / \psi$ production. In the UA1 experiment, it was not possible to separate the bottom-quark contribution.

Later, the CDF and D0 collaborations, when measuring $J / \psi$ production at the Fermilab Tevatron $(\sqrt{s}=1.8 \mathrm{TeV})$, were able to subtract the bottom-quark contribution from the total yield. Since also the yield of the $\chi_{c J}$ mesons decaying into $J / \psi$ mesons could be separated, a measurement of prompt $J / \psi$ production at large $p_{T}$ was possible. (We shall use the term prompt instead of direct to denote all $J / \psi$ mesons not originating from decays, i.e., $B$-meson or $\chi_{c J}$ decays.) The cross section of prompt inclusive $J / \psi$ production measured by CDF and D0 [4] turned out to be an order of magnitude larger than the prediction of the CSM. It is well known that, at large $p_{T}$, the dominant mechanism of light-quarkonium production is via the fragmentation of gluons and quarks, while the parton-fusion process is suppressed by powers $m^{2} / p_{T}^{2}$, where $m$ is the mass of the quarks bound in the quarkonium state. So, the fusion process, which is a higher-twist contribution, can contribute significantly only at extremely small $p_{T}$, which for the light quarkonia is in the soft region outside the perturbative regime. For $J / \psi$ production, this region, due to the larger charm-quark mass $m_{c}$, extends to larger $p_{T}$. But eventually, at $p_{T} \gg m_{c}$, the fragmentation process, although of higher order in $\alpha_{s}$ compared to the fusion process, can exceed the fusion process. In fact, it was pointed out by Braaten and Yuan [5] that, in addition to the parton-fusion contributions described by the CSM, fragmentation of gluons and charm quarks is an important source for large- $p_{T}$ mesons in hadron-hadron collisions.

$J / \psi$ production in high-energy ep collisions at HERA is dominated by photoproduction, where the electron is scattered by a small angle producing photons of almost zero virtuality. First experimental results in the kinematical region $30 \mathrm{GeV}<W<150 \mathrm{GeV}$, where $W$ is the $\gamma p$ centre-of-mass (CM) energy, have been presented by the $\mathrm{H} 1$ collaboration [6]. In this work, the inelastic process is analysed for the first time. Most of the events come from the elastic and diffractive processes. They can be separated by measuring the 
$J / \psi$ energy spectrum in terms of the inelasticity variable $\tilde{z}=p_{p} \cdot p_{J / \psi} / p_{p} \cdot p_{\gamma}$, with $p_{p}, p_{\gamma}$, and $p_{J / \psi}$ being the four-momenta of the proton, the photon, and the $J / \psi$ meson, respectively. For elastic/diffractive events, one has $\tilde{z} \approx 1$. A clean sample of inelastic events can be obtained by selecting events with $\tilde{z}<0.9$. Due to the limited integrated luminosity in the 1994 HERA operation, the inelastic $J / \psi$ production cross section $d \sigma / d p_{T}^{2}$ could only be measured for $p_{T}^{2}<6 \mathrm{GeV}^{2}$ [6]. In this range of $p_{T}^{2}$, the inelastic production proceeds predominantly through the fusion mechanism in the CSM. In fact, the experimental $p_{T}$ and $\tilde{z}$ distributions are very well described, as for both shape and absolute normalization, by the CSM next-to-leading-order (NLO) calculation of Krämer [7], using up-to-date NLO parton density functions (PDF's) of the proton.

As is well known, high-energy photoproduction proceeds through two distinct mechanisms: direct photoproduction, where the photon couples directly to the quarks, and resolved photoproduction, where the photon interacts through one of its partonic components, quarks or gluons, via hard scattering with the partonic constituents of the proton. For $J / \psi$ photoproduction within the CSM, the resolved-photon process is

$$
\gamma+p \rightarrow g_{\gamma}+g_{p} \rightarrow c \bar{c}\left[\underline{1},{ }^{3} S_{1}\right]+g \rightarrow J / \psi+g .
$$

This means that the gluon coming from the proton interacts with the gluon from the proton to create a ${ }^{3} S_{1}$ colour-singlet $c \bar{c}$ pair, which subsequently fuses into a $J / \psi$ meson. This process has been analysed [8] with the result that its contribution to the overall cross section in the CSM is small everywhere, except in the very-low- $\tilde{z}$ region. Contributions from the production and radiative decay of $\chi_{c J}$ states are of similar magnitude; see Ref. 88] for a review of these and other resolved-photoproduction mechanisms within the CSM.

Within the factorization approach, $J / \psi$ production can also proceed through colouroctet $c \bar{c}$ pairs. They appear for direct photoproduction as well as for resolved photoproduction. Colour-octet configurations which contribute to inelastic direct $J / \psi$ photoproduction are produced through the following subprocesses:

$$
\begin{aligned}
& \gamma+g \rightarrow c \bar{c}\left[\underline{8},{ }^{1} S_{0}\right]+g, \\
& \gamma+g \rightarrow c \bar{c}\left[\underline{8},{ }^{3} S_{1}\right]+g, \\
& \gamma+g \rightarrow c \bar{c}\left[\underline{8},{ }^{3} P_{J}\right]+g,
\end{aligned}
$$

where $J=0,1,2$. Other contributions, such as light-quark initiated ones, are strongly suppressed at HERA energies and can be safely neglected. Adopting the colour-octet matrix elements obtained through fits to prompt $J / \psi$ data from the Tevatron [9], one finds that colour-singlet and colour-octet contributions to inelastic direct photoproduction are of comparable size [10]. In the case of resolved photoproduction, the following colour-octet processes contribute:

$$
\begin{aligned}
& g_{\gamma}+g_{p} \rightarrow c \bar{c}\left[\underline{8},{ }^{1} S_{0}\right]+g, \\
& g_{\gamma}+g_{p} \rightarrow c \bar{c}\left[\underline{8},{ }^{3} S_{1}\right]+g, \\
& g_{\gamma}+g_{p} \rightarrow c \bar{c}\left[\underline{8},{ }^{3} P_{J}\right]+g .
\end{aligned}
$$


These are the same processes that contribute significantly to $J / \psi$ production in $p \bar{p}$ collisions at the Tevatron [9]. Their contribution to inelastic $J / \psi$ photoproduction has also been calculated [11] and found to be non-negligible. In fact, it is comparable to the cross section of colour-singlet direct photoproduction [11]. The distributions in the inelasticity variable $\tilde{z}$ are, however, quite different. The resolved-photon cross section (colour-singlet plus colour-octet) is dominant at small $\tilde{z}$, whereas the direct-photon contribution is large towards $\tilde{z}=1$.

For $\chi_{c J}$ production, the colour-octet channels are much more important. In the case of direct photoproduction, the production of colour-singlet $\chi_{c J}$ states via

$$
\gamma+g \rightarrow c \bar{c}\left[\underline{1},{ }^{3} P_{J}\right]+g \rightarrow \chi_{c J}+g
$$

is forbidden to leading order in $\alpha_{s}$, so that the colour-octet processes

$$
\begin{gathered}
\gamma+g \rightarrow c \bar{c}\left[\underline{8},{ }^{3} S_{1}\right]+g, \\
\gamma+q(\bar{q}) \rightarrow c \bar{c}\left[\underline{8},{ }^{3} S_{1}\right]+q(\bar{q}),
\end{gathered}
$$

where the colour-octet ${ }^{3} S_{1} c \bar{c}$ pair fuses into a physical $\chi_{c J}$ particle, are the dominant ones. Nevertheless, using the matrix elements obtained through fits to the Tevatron data [9:12.13], the contribution of the channels (5) is still small compared to the dominant CSM contribution to $J / \psi$ production [11]. The cross sections for resolved photoproduction of $\chi_{c J}$ mesons in the colour-octet channels have not yet been estimated.

All these results refer to $J / \psi$ production at $p_{T}$ values of order $m_{c}$, where we may expect the higher-twist contribution to be dominant. At sufficiently large $p_{T}$, the fragmentation process is supposed to be the dominant mechanism of $J / \psi$ photoproduction. First estimates of the fragmentation contribution to $J / \psi$ photoproduction have been presented in Ref. [14]; see also Ref. [15] for earlier work within the CSM. These authors considered, at leading order (LO), the fragmentation of charm quarks and gluons produced through the direct-photon processes $\gamma+q \rightarrow g+q$ and $\gamma+g \rightarrow c+\bar{c}$. They did not take into account resolved photoproduction, which is certainly important, too. In the case of $J / \psi$ production in $p \bar{p}$ collisions at the Tevatron, it is the analogous mechanism which makes the dominant contribution [12,13].

It is the purpose of this work to study $J / \psi$ photoproduction at large $p_{T}$ including all direct- and resolved-photoproduction channels at NLO. We shall focus our attention on the production via fragmentation. We shall consider the fusion mechanism only for comparison; for simplicity, we shall only include its dominant LO contribution, which arises from direct photoproduction in the CSM. The superposition of the NLO cross sections for the fusion and fragmentation mechanisms is left to future work.

The framework for our calculation of the NLO fragmentation cross section is similar to the one employed recently for the calculation of inclusive $D^{* \pm}$ photoproduction [16]. At large $p_{T}$, one may neglect the mass of the charm quark. The collinear singularities corresponding to the $\alpha_{s} \ln \left(p_{T}^{2} / m_{c}^{2}\right)$ terms of the massive-charm scheme, which is used, for example, for predictions at $p_{T} \lesssim m_{c}$, are then absorbed into the charm-quark PDF's and the fragmentation functions (FF's) in the same way as for the lighter $u, d$, and $s$ 
quarks. The FF's for the fragmentation of charm quarks and gluons into $J / \psi$ mesons must be known for this approach at the scale of production, which is usually taken to

be $\mu=m_{T}$, where $m_{T}=\sqrt{M_{J / \psi}^{2}+p_{T}^{2}}$ is the transverse mass of the $J / \psi$ meson with mass $M_{J / \psi} \approx 2 m_{c}$. These FF's can be computed at the starting scale $\mu_{0}=M_{J / \psi}$ in nonrelativistic QCD adopting as nonperturbative input the colour-singlet and colouroctet transition-matrix elements, which also enter the fusion approach. At larger scales $\mu \gg M_{J / \psi}$, the large logarithms in $\mu / M_{J / \psi}$ are resummed using the usual Altarelli-Parisi evolution equations. At the starting scale, only the fragmentation of gluons and charm quarks need to be included, while the light-quark contributions are negligible. The latter will be generated at higher scales via evolution.

The outline of our work is as follows. In Section 2, we shall shortly describe the formalism for the perturbative FF's and discuss the transition from massless to massive factorization used also in our previous work [16. In Section 3, we shall present our NLO predictions for the various fragmentation contributions to the cross section of $J / \psi$ photoproduction in ep collisions at HERA as functions of $p_{T}$ and rapidity $y$, and compare them with the LO CSM results based on the $c \bar{c}$ fusion mechanism. Specifically, we shall distinguish between direct-photon and resolved-photon, prompt and non-prompt (only via $\chi_{c J}$ states), colour-singlet and colour-octet fragmentation production. Our conclusions will be summarized in Section 4 .

\section{Charmonium fragmentation functions}

In this section, we shall describe the assumptions underlying the massless approach, which is very similar to that in our work on inclusive $D^{*}$ production [16]. For the reader's convenience, we shall repeat here the basic ideas developed in Ref. [16]. As already mentioned in the Introduction, two mechanism contribute to the photoproduction of charm quarks in ep collisions: $(i)$ In the direct photoproduction mechanism, the photon couples directly to the quarks, which besides the massless $u, d$, and $s$ quarks, also include the massless charm quark. In this case, at least in the LO processes, no spectator particles travel along the momentum direction of the photon. (ii) In the resolved photoproduction mechanism, the photon splits up into fluxes of $u, d, s, c$ quarks and gluons, which then interact with the partons coming from the proton leading to the production of quarks, including charm quarks, and gluons at large $p_{T}$. The contributing parton-level processes are the same as in the case of $J / \psi$ production in hadron-hadron collisions. The quarks and gluons coming out of the photon are accompanied by a spectator jet, which also travels in the photon direction. Therefore, the $\gamma p$ cross section depends not only on the PDF's of the proton but also on those of the photon. The main difference with respect to the usually considered massive-charm scheme, which to our knowledge has not yet been worked out for $J / \psi$ production via fragmentation, is that the charm quark also contributes via the PDF's of the proton and the photon, i.e., charm is already an active flavour in the initial state. Compared to $D^{* \pm}$ production, where the $D^{* \pm}$ mesons are almost exclusively produced via charm-quark fragmentation, in $J / \psi$ production the fragmentation process $g \rightarrow J / \psi+X$ is 
very important as well, as we shall see in the next section. The massless-charm approach is justified if a large scale is governing the production process. In our case, this is the transverse momentum of the $J / \psi$ meson, with $p_{T} \gg M_{J / \psi}$. In this region, non-singular mass terms are suppressed in the cross section by powers of $m_{c} / p_{T}$. The important mass terms appear if the virtuality of the charm quark is small. This occurs in the initial state if the charm quark is emitted from the proton or photon, and in the final state if the partons emit a $c \bar{c}$ pair. These two contributions lead to the $\alpha_{s} \ln \left(p_{T}^{2} / m_{c}^{2}\right)$ terms in the massive scheme. In our massless approach, they are summed into the scale-dependent proton and photon PDF's and $J / \psi$ FF's.

To obtain the final $J / \psi$ production cross sections in NLO, the following steps are taken. (i) The NLO hard-scattering cross sections for the direct- and resolved-photon processes are calculated in the massless approximation with $n_{f}=4$ active flavours [17. The collinear singularities are subtracted according to the $\overline{\mathrm{MS}}$ scheme. Since the charm quark is taken to be massless, the singularities from its splittings are subtracted as well. (ii) The charm quark is accommodated in the PDF's of the proton and photon as a light flavour. The finite mass of the charm quark is taken into account by including it in the evolution of the PDF's in such a way that these are only non-vanishing above a scale set by its mass. (iii) The FF's characterize the hadronization of the massless partons, including the charm quark, into $c \bar{c}$ bound states, i.e., the $J / \psi$ and $\chi_{c J}$ mesons. Similarly to the fragmentation into light mesons, these FF's are non-perturbative input and must be determined by experiment, for example from the cross section of inclusive $J / \psi$ and $\chi_{c J}$ production in hadron-hadron collisions. This information determines the FF's at some starting scale, $\mu_{0}$.

An alternative methods is to calculate, within perturbative QCD, universal starting conditions for the FF's at a scale $\mu_{0}$ of order $M_{J / \psi}$. This allows one to include in a straightforward manner the colour-octet contributions with parameters related to the ones obtained from the analysis of colour-octet fusion.

The structure of the perturbative FF's is based on the general factorization analysis of the production and decay of heavy quarkonia by Bodwin, Braaten, and Lepage [18]. This factorization formalism allows for the FF's of charmonium production to be factorized into short-distance coefficients that describe the production rate of a $c \bar{c}$ pair within a region of size $1 / m_{c}$, and long-distance factors that contain the non-perturbative dynamics responsible for the formation of the bound state $H$ containing the $c \bar{c}$ pair. The FF, at factorization scale $\mu$, for a parton $a$ to fragment into the charmonium state $H$ with longitudinal momentum fraction $z$ can be written as

$$
D_{a}^{H}(z, \mu)=\sum_{n} d_{a}^{n}(z, \mu)\left\langle 0\left|\mathcal{O}_{n}^{H}\right| 0\right\rangle
$$

where $n$ labels different colour components (singlet or octet) and spin quantum numbers to be specified later. $\mathcal{O}_{n}^{H}$ are the local four-fermion operators defined in Ref. 18 in terms of the fields of nonrelativistic QCD. The short-distance coefficients $d_{a}^{n}(z, \mu)$ can be computed at the starting scale $\mu_{0}$ using perturbation theory in $\alpha_{s}\left(\mu_{0}\right)$. The dependence on the observed charmonium state $H$ only appears in the long-distance factors $\left\langle 0\left|\mathcal{O}_{n}^{H}\right| 0\right\rangle$. 
As input for our analysis, we need the short-distance factors $d_{a}^{n}\left(z, \mu_{0}\right)$ and matrix elements $\left\langle 0\left|\mathcal{O}_{n}^{H}\right| 0\right\rangle$ for the following transitions: $g \rightarrow J / \psi, g \rightarrow \chi_{c J}, c \rightarrow J / \psi$, and $c \rightarrow \chi_{c J}$, which we shall take from the literature. In the case of the gluon FF's into $J / \psi$ and $\chi_{c J}$ mesons, we use the results of Refs. [5] and [19], respectively. The corresponding charm-quark FF's may be found in Refs. [20] and [21], respectively.

In order to understand our final results, it is necessary to know the relative importance of the various terms labelled $n$ in Eq. (6). On the one hand, this is determined by the magnitude of the short-distance factors $d_{a}^{n}(z, \mu)$ and, on the other hand, by the relative size of the various matrix elements $\left\langle 0\left|\mathcal{O}_{n}^{H}\right| 0\right\rangle$. The size of the latter may be estimated by how they scale with the relative velocity $v$ of the charm quarks inside the charmonium state. The magnitude of the $d_{a}^{n}(z, \mu)$ functions is controlled by the lowest order of $\alpha_{s}$ that appears in $d_{a}^{n}(z, \mu)$. Thus, to determine the relative importance of the terms in Eq. (6), one needs to know the scaling in $v$ of the matrix elements and the order in $\alpha_{s}$ of their coefficients.

Let us start with the gluon FF into $J / \psi$, which is a ${ }^{3} S_{1} c \bar{c}$ state. Keeping only the leading terms, Eq. (6) takes the form

$$
D_{g}^{J / \psi}\left(z, \mu_{0}\right)=\frac{\alpha_{s}^{3}\left(\mu_{0}\right)}{m_{c}^{3}} d_{g}^{\left[1,{ }^{3} S_{1}\right]}(z)\left\langle 0\left|\mathcal{O}_{1}^{J / \psi}\left({ }^{3} S_{1}\right)\right| 0\right\rangle+\frac{\alpha_{s}\left(\mu_{0}\right)}{m_{c}^{3}} d_{g}^{\left[{ }^{[},{ }^{3} S_{1}\right]}(z)\left\langle 0\left|\mathcal{O}_{8}^{J / \psi}\left({ }^{3} S_{1}\right)\right| 0\right\rangle,
$$

where $d_{g}^{\left[1,{ }^{3} S_{1}\right]}(z)$ and $d_{g}^{\left[8^{3}, S_{1}\right]}(z)$ [5] are dimensionless functions listed in the Appendix. The first term in Eq. (7) corresponds to the colour-singlet contribution leading in $v$. Its matrix element $\left\langle 0\left|\mathcal{O}_{1}^{J / \psi}\left({ }^{3} S_{1}\right)\right| 0\right\rangle$ is proportional to the probability for the formation of a $J / \psi$ meson from a pointlike $c \bar{c}$ pair in a color-singlet ${ }^{3} S_{1}$ state. It is related to the $J / \psi$ radial wave function at the origin, $R_{J / \psi}(0)$, by [22]

$$
\left\langle 0\left|\mathcal{O}_{1}^{J / \psi}\left({ }^{3} S_{1}\right)\right| 0\right\rangle=\frac{9}{2 \pi}\left|R_{J / \psi}(0)\right|^{2},
$$

up to corrections of relative order $v^{4}$, and may be extracted from the partial width of the leptonic decay $J / \psi \rightarrow \ell^{+} \ell^{-}$. Following Ref. [22], we use $\left\langle 0\left|\mathcal{O}_{1}^{J / \psi}\left({ }^{3} S_{1}\right)\right| 0\right\rangle=1.13 \mathrm{GeV}^{3}$, which includes the $O\left(\alpha_{s}\right)$ correction to the leptonic width. The leading contribution to the short-distance coefficient $d_{g}^{\left[1,{ }^{3} S_{1}\right]}(z)$ comes from the parton process $g^{*} \rightarrow c \bar{c} g$, where $g^{*}$ denotes a virtual gluon, and is of order $\alpha_{s}^{3}$. The matrix element $\left\langle 0\left|\mathcal{O}_{1}^{J / \psi}\left({ }^{3} S_{1}\right)\right| 0\right\rangle$ is of order $v^{3}$, so that the contribution to the gluon $\mathrm{FF}$ is of order $\alpha_{s}^{3} v^{3}$. All other matrix elements in the expansion in Eq. (77) are of higher order in $v^{2}$ and would, therefore, be neglected in the colour-singlet approximation. However, in the charmonium system, $v^{2}$ is not actually small; typically one has $v^{2} \approx 1 / 4$, so that not all matrix elements that are formally suppressed by powers of $v^{2}$ may be neglected in practice. Of particular importance is the matrix element $\left\langle 0\left|\mathcal{O}_{8}^{J / \psi}\left({ }^{3} S_{1}\right)\right| 0\right\rangle$ because it has a short-distance factor of order $\alpha_{s}$, which which arises from the parton process $g^{*} \rightarrow c \bar{c}$. The corresponding matrix element $\left\langle 0\left|\mathcal{O}_{8}^{J / \psi}\left({ }^{3} S_{1}\right)\right| 0\right\rangle$ is of order $v^{4}$. We take its numerical value to be $\left\langle 0\left|\mathcal{O}_{8}^{J / \psi}\left({ }^{3} S_{1}\right)\right| 0\right\rangle=$ $0.014 \mathrm{GeV}^{3}$ [22]. Thus, the suppression factor $v^{4}$ is compensated by the factor $1 / \alpha_{s}^{2}$.

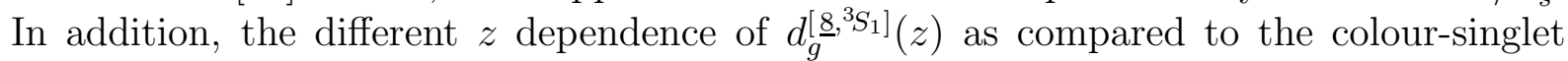


short-distance coefficient $\left.d_{g}^{[1},{ }^{3} S_{1}\right](z)$ enhances the relative importance of the colour-octet contribution.

The gluon FF's into the ${ }^{3} P_{J}$ charmonia $\chi_{c J}$ have been worked out in Ref. [19] and take the form

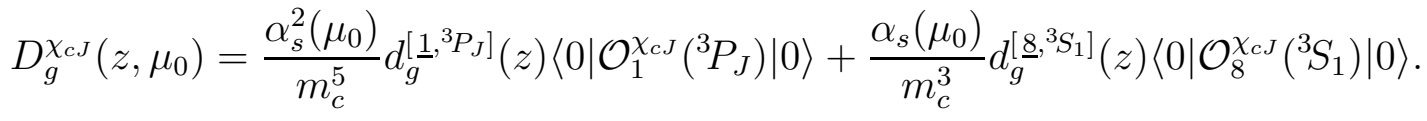

The colour-singlet matrix element $\left\langle 0\left|\mathcal{O}_{1}^{\chi_{c J}}\left({ }^{3} P_{J}\right)\right| 0\right\rangle$ is related to the derivative of the nonrelativistic radial wave function of the $P$-wave states at the origin, $R_{\chi_{c}}^{\prime}(0)$, by [22]

$$
\left\langle 0\left|\mathcal{O}_{1}^{\chi_{c J}}\left({ }^{3} P_{J}\right)\right| 0\right\rangle=(2 J+1) \frac{9}{2 \pi}\left|R_{\chi_{c}}^{\prime}(0)\right|^{2},
$$

up to corrections of relative order $v^{4}$. This parameter can be determined phenomenologically from the annihilation rates of the $\chi_{c J}$ mesons. Since both matrix elements are of the same order in $v$ and the colour-octet term has one power of $\alpha_{s}$ less, it is expected to dominate. According to Refs. [19] and [22], the matrix elements take the values

$$
\begin{aligned}
& \left\langle 0\left|\mathcal{O}_{1}^{\chi_{c 0}}\left({ }^{3} P_{0}\right)\right| 0\right\rangle=\frac{1}{2 J+1}\left\langle 0\left|\mathcal{O}_{1}^{\chi_{c J}}\left({ }^{3} P_{J}\right)\right| 0\right\rangle=0.0862 \mathrm{GeV}^{5} \\
& \left\langle 0\left|\mathcal{O}_{8}^{\chi_{c 0}}\left({ }^{3} S_{1}\right)\right| 0\right\rangle=\frac{1}{2 J+1}\left\langle 0\left|\mathcal{O}_{8}^{\chi_{c J}}\left({ }^{3} S_{1}\right)\right| 0\right\rangle=0.0076 \mathrm{GeV}^{3} .
\end{aligned}
$$

In the evaluation of Eq. (11]), we have used $2 m_{c}=M_{J / \psi}=3.09688 \mathrm{GeV}$ [23]. The value of the colour-octet matrix element in Eq. (12) is larger than the most recent value obtained from $B$-meson decays 12 .

We now turn to charm-quark fragmentation. The LO formula for the $c \rightarrow J / \psi \mathrm{FF}$ has been found in Ref. [20] and takes the form

$$
\left.D_{c}^{J / \psi}\left(z, \mu_{0}\right)=\frac{\alpha_{s}^{2}\left(\mu_{0}\right)}{m_{c}^{3}} d_{c}^{[1},{ }^{3} S_{1}\right](z)\left\langle 0\left|\mathcal{O}_{1}^{J / \psi}\left({ }^{3} S_{1}\right)\right| 0\right\rangle+\frac{\alpha_{s}^{2}\left(\mu_{0}\right)}{m_{c}^{3}} d_{c}^{\left[\underline{8},{ }^{3} S_{1}\right]}(z)\left\langle 0\left|\mathcal{O}_{8}^{J / \psi}\left({ }^{3} S_{1}\right)\right| 0\right\rangle,
$$

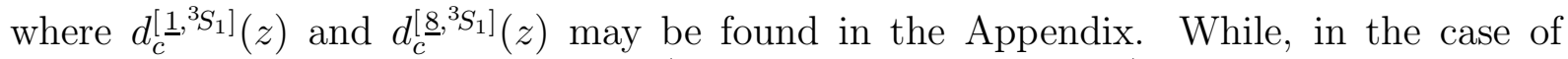
$g \rightarrow J / \psi$, the $v^{4}$ suppression of $\left\langle 0\left|\mathcal{O}_{8}^{J / \psi}\left({ }^{3} S_{1}\right)\right| 0\right\rangle$ relative to $\left\langle 0\left|\mathcal{O}_{1}^{J / \psi}\left({ }^{3} S_{1}\right)\right| 0\right\rangle$ is compensated by the fact that the colour-octet coefficient is enhanced by a factor $1 / \alpha_{s}^{2}$ relative to the colour-singlet one, such a compensation does not occur in Eq. (13). In fact, the second term in Eq. (13) only amounts to about $0.12 \%$ of the first one.

The $c \rightarrow \chi_{c J}$ FF's are given by [21]

$$
\left.D_{c}^{\chi_{c J}}\left(z, \mu_{0}\right)=\frac{\alpha_{s}^{2}\left(\mu_{0}\right)}{m_{c}^{5}} d_{c}^{[1,}{ }^{3} P_{J}\right](z)\left\langle 0\left|\mathcal{O}_{1}^{\chi_{c J}}\left({ }^{3} P_{J}\right)\right| 0\right\rangle+\frac{\alpha_{s}^{2}\left(\mu_{0}\right)}{m_{c}^{3}} d_{c}^{\left[{ }^{8},{ }^{3} S_{1}\right]}(z)\left\langle 0\left|\mathcal{O}_{8}^{\chi_{c J}}\left({ }^{3} S_{1}\right)\right| 0\right\rangle,
$$

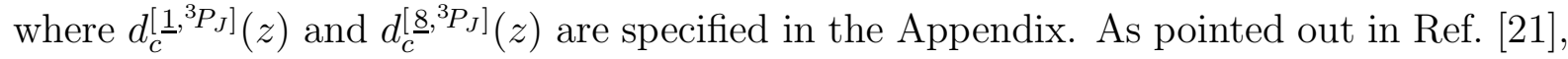
the colour-octet term in Eq. (14) is minuscule. Although the minimum invariant mass 
of the fragmenting charm quark is $3 m_{c}$, for consistency with the gluon FF's, we choose $\mu_{0}=2 m_{c}$ in Eqs. (13) and (14).

For the calculation of the $J / \psi$ production cross sections, we need the FF's at the factorization scale $\mu \gg M_{J / \psi}$. Then, large logarithms in $\mu / M_{J / \psi}$ appear, which have to be resummed. This is achieved by using the Altarelli-Parisi equations,

$$
\frac{\mu^{2} d}{d \mu^{2}} D_{a}^{H}(z, \mu)=\sum_{b} \int_{z}^{1} \frac{d x}{x} P_{b a}^{(T)}\left(\frac{z}{x}, \alpha_{s}(\mu)\right) D_{b}^{H}(x, \mu),
$$

where

$$
P_{b a}^{(T)}\left(z, \alpha_{s}(\mu)\right)=\frac{\alpha_{s}(\mu)}{2 \pi} P_{b a}^{(0, T)}(z)+\left(\frac{\alpha_{s}(\mu)}{2 \pi}\right)^{2} P_{b a}^{(1, T)}(z)+O\left(\alpha_{s}^{3}\right)
$$

are the timelike splitting functions of parton $a$ into parton $b$. In our NLO analysis, we include the splitting functions through order $\alpha_{s}^{2}$ [24], while in our LO analysis we truncate Eq. (16) after the first term. The initial conditions $D_{a}^{H}\left(z, \mu_{0}\right)$ for $a=g, c$ and $H=J / \psi, \chi_{c J}$ have been specified above. Due to charge-conjugation invariance, we have $D_{\bar{c}}^{H}\left(z, \mu_{0}\right)=D_{c}^{H}\left(z, \mu_{0}\right)$. The initial light-quark FF's are set equal to zero. They are generated at larger scales $\mu$ via Eq. (15). However, we expect their effect to be small. The splitting functions $P_{b a}^{(T)}\left(z, \alpha_{s}(\mu)\right)$ are for massless quarks throughout.

In Ref. [25], an improved version of Eq. (15), which respects the phase-space constraint $D_{a}^{H}(z, \mu)=0$ for $z<M_{H}^{2} / \mu^{2}$, has been suggested. In Ref. [26], it was found that the numerical effect of this improvement is not crucial at large scales. Since we are mainly interested in the fragmentation production of $J / \psi$ mesons at large $p_{T}$, for simplicity, we shall stick to Eq. (15) for the time being, leaving the implementation of the generalized evolution equations to future work.

As in our recent work on $D^{* \pm}$ photoproduction [16], we start from the NLO hardscattering cross sections calculated in the $\overline{\mathrm{MS}}$ scheme with massless flavours, and slightly modify the factorization scheme for the collinear singularities associated with final-state charm quarks. Specifically, whenever final-state collinear singularities are subtracted from the hard-scattering cross sections, we substitute $P_{c a}^{(0, T)}(z) \ln \left(s / \mu^{2}\right) \rightarrow P_{c a}^{(0, T)}(z) \ln \left(s / \mu^{2}\right)-$ $d_{c a}(z)$, where $P_{c a}^{(0, T)}(z)$ are the LO timelike $a \rightarrow c$ splitting functions of Eq. (16) and the $d_{c a}(z)$ functions may be found in Ref. [16]. Here, $a=g, c, q$, where $q$ stands for

the first three quark flavours. With this procedure, the factorization of the final-state collinear singularities associated with the charm quark is adjusted so as to match the finite- $m_{c}$ calculation. This is equivalent to the matching approach proposed in Ref. [27] between the massless-charm calculation in connection with the perturbative FF's and the massive-charm calculation without FF's.

\section{Results}

This section consists of three parts. Firstly, we shall specify our assumptions concerning the proton and photon PDF's as well as the equivalent photon approximation. Secondly, we shall present our predictions for the particular range of $\gamma p \mathrm{CM}$ energies $W$ that is used 
in the $\mathrm{H} 1$ analysis of inclusive $J / \psi$ production [6], namely, $30 \mathrm{GeV}<W<150 \mathrm{GeV}$. In this subsection, we shall compare the results for the various channels of interest: prompt $J / \psi$ photoproduction versus non-prompt $J / \psi$ production originating from $\chi_{c J}$ photoproduction with subsequent $\chi_{c J} \rightarrow J / \psi+\gamma$ decay; colour-singlet channel versus colour-octet channel; direct photoproduction versus resolved photoproduction. In the third part, we consider the high- $W$ range, $150 \mathrm{GeV}<W<280 \mathrm{GeV}$, where the fragmentation contribution is greatly increased relative to the fusion contribution.

\subsection{Input information}

For the calculation of the cross section $d^{2} \sigma / d y_{\text {lab }} d p_{T}$, we adopt the present HERA conditions, where $E_{p}=820 \mathrm{GeV}$ protons collide with $E_{e}=27.5 \mathrm{GeV}$ positrons in the laboratory frame, so that $\sqrt{s}=2 \sqrt{E_{p} E_{e}}=300 \mathrm{GeV}$ is available in the $\mathrm{CM}$ frame. We take the rapidity $y_{l a b}$ to be positive in the proton flight direction. The quasi-real-photon spectrum is described in the Weizsäcker-Williams approximation by the formula

$$
f_{\gamma / e}(x)=\frac{\alpha}{2 \pi}\left[\frac{1+(1-x)^{2}}{x} \ln \frac{Q_{\max }^{2}}{Q_{\min }^{2}}+2 m_{e}^{2} x\left(\frac{1}{Q_{\max }^{2}}-\frac{1}{Q_{\min }^{2}}\right)\right],
$$

where $x=E_{\gamma} / E_{e}=W^{2} / s, Q_{m i n}^{2}=m_{e}^{2} x^{2} /(1-x), \alpha$ is Sommerfeld's fine-structure constant, and $m_{e}$ is the electron mass. We consider the case where the final-state electron is not tagged, so that $Q_{\max }^{2}=4 \mathrm{GeV}^{2}$. The $\gamma p$ energy interval $30 \mathrm{GeV}<W<150 \mathrm{GeV}$ $(150 \mathrm{GeV}<W<280 \mathrm{GeV})$ considered in Section $3.2(3.3)$ corresponds to $0.010<x<$ $0.249(0.249<x<0.869)$.

We work at NLO in the $\overline{\mathrm{MS}}$ scheme with $n_{f}=4$ flavours. As for the proton and photon PDF's, we use set CTEQ4M [28], with $\Lambda \frac{(4)}{\mathrm{MS}}=296 \mathrm{MeV}$, and set GRV HO [29] after converting it from the $\mathrm{DIS}_{\gamma}$ scheme to the $\overline{\mathrm{MS}}$ scheme. We identify the factorization scales associated with the proton, photon and final-state hadron and collectively denote them by $M_{f}$. For $M_{f}$ and the renormalization scale $\mu$, we choose $\mu=M_{f}=m_{T}$, where $m_{T}$ is the $J / \psi$ transverse mass defined above. We take the starting scale of the FF's to be $\mu_{0}=2 m_{c}$. We calculate $\alpha_{s}(\mu)$ from the two-loop formula with $\Lambda \frac{(4)}{\mathrm{MS}}$ equal to the value used in the proton PDF's. Unfortunately, the NLO correction to the perturbative coefficient functions discussed above, which, strictly speaking, ought to be included as well, are not yet available. However, in analogy to the genuine NLO corrections to $J / \psi$ photoproduction at HERA in the CSM, which are only at the $20 \%$ level, these are likely to be moderate as well.

The LO results which enter the QCD-correction $(K)$ factors are calculated consistently, i.e., using the one-loop formula for $\alpha_{s}(\mu)$, the CTEQ4L [28] proton PDF's, with $\Lambda \frac{(4)}{\mathrm{MS}}=$ $236 \mathrm{MeV}$, and the GRV LO photon PDF's, and evolving the FF's with the LO splitting functions. In all calculations, we employ the numerical values of the matrix elements specified above. 


\subsection{Results for the low- $W$ range}

In this subsection, we investigate the NLO cross section $d^{2} \sigma / d y_{l a b} d p_{T}$ of inclusive $J / \psi$ photoproduction at HERA, integrated over $30 \mathrm{GeV}<W<150 \mathrm{GeV}$ as in the H1 experiment [6]. In order to analyze the $p_{T}$ dependence of the cross section in the central region of the detector, we integrate $d^{2} \sigma / d y_{l a b} d p_{T}$ over $-1.5<y_{l a b}<1$. The result is displayed in Figs. 1(a)-(c) for direct and resolved photoproduction and their sum, respectively. In each case, we show how the total fragmentation contributions (solid lines) are composed of the respective prompt colour-singlet (dashed lines), prompt colour-octet (dot-dashed lines), and non-prompt (dot-dot-dashed lines) contributions. For comparison, we also show the LO direct-photon contribution of the CSM (dotted lines). We have to bear in mind that the latter is enhanced by typically $20 \%$ due to genuine NLO QCD corrections to the parton-fusion cross section [7]. However, we implicitly include the bulk of the QCD corrections, namely, those to the leptonic $J / \psi$ decay width, which affect the value of $\left\langle 0\left|\mathcal{O}_{1}^{J / \psi}\left({ }^{3} S_{1}\right)\right| 0\right\rangle$ extracted from experiment [22]. For consistency with the fragmentation contribution, we also choose $\mu=M_{f}=m_{T}$ in the fusion contribution. We do not take into account the resolved-photon CSM contribution, which appreciably contributes only at very low $\tilde{z}$, i.e., at low $p_{T}$ and large $y_{\text {lab }}$ [8]. A dedicated study of the low- $\tilde{z}$ range, which we intend to do in the future, should also consider this contribution.

As anticipated in the Introduction, both in the direct- and resolved-photon channels, the fragmentation cross sections fall off less steeply with $p_{T}$ than the fusion cross section. In all cases, we observe that the prompt colour-octet contribution is most significant and dominates the large- $p_{T}$ behaviour. The non-prompt cross section is always smaller than the prompt cross section. In the sum of the direct- and resolved-photon channels, their ratio ranges from 2.8 at $p_{T}=3 \mathrm{GeV}$ to 4.3 at $p_{T}=15 \mathrm{GeV}$. We emphasize that, in the low- $p_{T}$ range, the resolved-photon component, which was previously neglected [14, is considerably more important than the direct-photon component, by a factor of 18 (2.3) at $p_{T}=3 \mathrm{GeV}(10 \mathrm{GeV})$. The two components cross over at $p_{T} \approx 15 \mathrm{GeV}$. Notice also that, even for $p_{T} \lesssim 6 \mathrm{GeV}$, the resolved-photon component is comparable to the fusion contribution; for $p_{T}>7 \mathrm{GeV}$, it exceeds the fusion contribution. In the resolved-photon contribution, the prompt colour-singlet part is negligibly small compared to the prompt colour-octet part. This is caused by the dominance of $g \rightarrow J / \psi+X$ in the resolved-photon cross section and the suppression of colour-singlet gluon fragmentation mentioned above. In the direct-photon cross section, this hierarchy is reduced since $c \rightarrow J / \psi+X$ is more important.

In general, rapidity distributions allow for a more specific investigation of the individual contributions than transverse-momentum distributions. In Figs. 2(a)-(c), the $y_{\text {lab }}$ dependence of $d^{2} \sigma / d y_{l a b} d p_{T}$ is displayed at $p_{T}=5$ and $10 \mathrm{GeV}$ for the same cases as in Figs. I (a)-(c). While the direct-photon component peaks in the same $y_{\text {lab }}$ region as the fusion contribution, the resolved-photon cross section dominantly contributes in the forward direction, with a maximum at about $y_{l a b}=2.5$. As might be inferred from Figs. 1 1 (a)-(c), the prompt colour-octet contribution is dominant; in fact, this is true over the full $y_{\text {lab }}$ range. We observe that the $y_{l a b}$ spectra of the prompt colour-singlet channel, although 
suppressed, have a shape similar to the fusion contribution and are peaked at significantly smaller $y_{\text {lab }}$ values than the other fragmentation channels. At large $p_{T}$, the dominant $J / \psi$ production mechanism in $p \bar{p}$ collisions at the Tevatron is colour-octet gluon fragmentation [30]. In order for HERA experiments to probe this component, it will be necessary to take data in the very forward direction, where the resolved-photon colour-octet contribution is most significant. It should be clear that the colour-octet contributions shown in Figs. 2(a)-(c) are proportional to the corresponding matrix elements specified in Section 2, which are extracted from fits to Tevatron data. So, in order to substantiate (or question) the huge prompt colour-octet $J / \psi$ contribution that is apparently indispensable to reconcile the Tevatron data with theory, it would be desirable to collect data in the very forward region at HERA, which has not yet been explored by the H1 and ZEUS collaborations.

As mentioned in the Introduction, the present analysis extends the previous work [14] on $J / \psi$ fragmentation production at HERA in two important respects. For one thing, we include the resolved-photon contribution, which was not mentioned in Ref. [14. On the other hand, we work at NLO, while the analysis of Ref. [14] proceeds at LO. Above, we have stressed the significance of the resolved-photon contribution, which is nicely illustrated in Figs. 1(b) and 2(b). In the following, we shall assess the impact of the NLO corrections on the various fragmentation contributions. To this end, we consistently repeat the analysis of Figs. 1(a)-(c) at LO, as described in Section 2, and plot the resulting NLO to LO ratios in Figs. B(a)-(c). In the case of direct photoproduction considered in

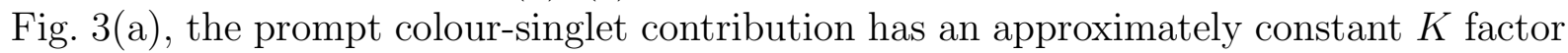
of about 0.6 , while the prompt colour-octet and non-prompt contributions have diminishing $K$ factors only at $p_{T}$ values below 6.5 and 4 , respectively. In the case of resolved photoproduction addressed in Fig. (3)(b), the $K$ factors of the various channels are always significantly larger than unity, and the overall $K$ factor ranges between 2 and 2.4. In both the direct- and resolved-photon cases, the $K$ factors of the non-prompt contributions take extraordinarily large values in the upper $p_{T}$ range. Detailed investigation reveals that this enhancement essentially originates from the lower edge of the kinematically allowed $y_{\text {lab }}$ range. However, since, at large $p_{T}$, these contributions are greatly suppressed relative to the prompt colour-octet contributions, this is inconsequential for the superposition of all channels, which is experimentally observable. In fact, the overall $K$ factor, which is shown as the solid line in Fig. 通(c), is well behaved, ranging between 1.6 and 1.8 in the $p_{T}$ range considered.

At this point, we should comment on the LO analysis of direct $J / \psi$ photoproduction via fragmentation at HERA reported in Ref. [14]. Similarly to the present study, the authors of Ref. [14 included the prompt, non-prompt, colour-singlet, and colour-octet contributions. However, they did not convolute the $\gamma p$ cross section with the WeizsäckerWilliams distribution, but considered fixed photon energies instead. There is an overall factor of 18 missing on the right-hand side of Eq. (3) in Ref. [14], which is to describe the fusion cross section. In the case of fragmentation production, a meaningful quantitative comparison is impossible, since the input values of the poorly known colour-octet matrix elements are not to be found in Ref. [14]. 
One should bear in mind that all results presented in this subsection refer to the low$W$ range $30 \mathrm{GeV}<W<150 \mathrm{GeV}$. We expect that the resolved-photon contribution will be enhanced relative to the fusion contribution if $W$ is increased. In the next subsection, this issue will be addressed in some detail.

\subsection{Results for the high- $W$ range}

In order to test the predictions for $J / \psi$ photoproduction via fragmentation at HERA that follow from the theoretical analysis of the Tevatron data, it is useful to find regions of phase space where the fusion mechanism is suppressed. As we have seen in Figs. 2(a)(c), this happens, for instance, in the very forward region of the detector. But even in the central region, $-1.5<y_{l a b}<1$, to which previous measurements of charmed-meson photoproduction at HERA were confined [31], fragmentation production may be enhanced with respect to the fusion mechanism by selecting events with high photon energy. This is demonstrated in Fig. 4 , where the $W$ dependence of $d^{2} \sigma / d W d p_{T}$ is shown for $p_{T}=5$ and $10 \mathrm{GeV}$. While the $W$ distribution of the direct-photon contribution has a shape similar to that of the fusion contribution, with a maximum at low to intermediate $W$, the resolved-photon contribution tends to be monotonically increasing with increasing $W$. As a consequence, even at low $p_{T}$, fragmentation production becomes more important than the fusion mechanism beyond some point in $W$. At $p_{T}=5$, the cross-over takes place at about $W=125 \mathrm{GeV}$, which corresponds to $x=0.17$ in Eq. (17).

Guided by this observation, we repeat the analysis of Fig. 11(c) integrating over $150 \mathrm{GeV}$ $<W<280 \mathrm{GeV}$, instead of $30 \mathrm{GeV}<W<150 \mathrm{GeV}$, and plot the outcome in Fig. 5. As expected, this leads to a significant increase of the fragmentation contribution, which mainly originates from the resolved-photon part. On the other hand, the fusion contribution is only moderately increased at $p_{T} \gtrsim 4 \mathrm{GeV}$, while it is even decreased at lower values of $p_{T}$. In conclusion, over the entire $p_{T}$ range, the fragmentation contribution is now more than twice as large as the fusion contribution; at $p_{T} \gtrsim 11 \mathrm{GeV}$, the fusion contribution falls short of the fragmentation contribution by more than one order of magnitude.

At HERA, it should also be possible to detect $J / \psi$ mesons in the very forward direction, almost up to $y_{l a b}=3.5$ [32]. This would offer the opportunity to render the fragmentation to fusion ratio even more favourable. Thus, in Fig. 6, we investigate how the results for $150 \mathrm{GeV}<W<280 \mathrm{GeV}$ in Fig. 5 are modified if the $y_{\text {lab }}$ interval is shifted from $-1.5<y_{l a b}<1$ to $1<y_{l a b}<3.5$. We observe that the fragmentation contribution is then enhanced, almost by a factor of two at large $p_{T}$. But, what is even more significant, the fusion contribution is reduced by one to two orders of magnitude. As a consequence, the fragmentation to fusion ratio is dramatically increased, to values in excess of 200 throughout the entire $p_{T}$ range. We conclude, that the kinematical region of large $W$ and large $y_{l a b}$ is an ideal place to probe at HERA the anomalously large inclusive $J / \psi$ yield observed at the Tevatron.

It has become customary to present experimental data on inclusive $J / \psi$ production in terms of the inelasticity variable $\tilde{z}$ defined in the Introduction, instead of using $y_{l a b}$. The 
relation between $\tilde{z}$ and $y_{l a b}$ reads

$$
\tilde{z}=\frac{2 E_{p} m_{T}}{W^{2}} e^{-y_{l a b}},
$$

where $E_{p}$ is the proton energy in the laboratory system. Thus, the kinematical range of interest corresponds to small values of $\tilde{z}$. This is in line with Ref. [14, where the kinematical cut $\tilde{z}<0.5$ has been proposed to enhance the fragmentation contribution relative to the fusion contribution. We have recently assessed, in a NLO analysis [33], the logistics of enhancing via appropriate acceptance cuts the colour-octet contribution to $J / \psi$ production via fragmentation at HERA.

\section{Conclusions}

In addition to the well-known parton-fusion mechanism, $J / \psi$ mesons may also be produced via the fragmentation of final-state partons. The latter mechanism is expected to be the dominant source of $J / \psi$ mesons at sufficiently large $p_{T}$. In this paper, we studied the photoproduction of $J / \psi$ mesons at large $p_{T}$ via fragmentation at HERA.

Our calculation was performed at NLO in the QCD-improved parton model with $n_{f}=4$ massless quark flavours, i.e., the charm quark was assumed to be an active flavour inside the proton and the resolved photon. We employed charm and gluon FF's which were perturbatively calculated at a fragmentation scale of order $M_{J / \psi}$ in nonrelativistic QCD, and evolved them in $z$ space to the characteristic scale of the considered process, of order $p_{T}$, using NLO Altarelli-Parisi splitting functions. At the same time, we adjusted the factorization scheme of the collinear singularities connected with final-state charm quarks so as to match the corresponding calculation with massive charm quarks. In this way, large logarithms of the type $\alpha_{s} \ln \left(p_{T}^{2} / M_{J / \psi}^{2}\right)$, which would be present in the massive calculation and render it unreliable at large $p_{T}$, are removed from the hard-scattering cross sections and properly resummed. We included prompt $J / \psi$ fragmentation production as well as $\chi_{c J}$ fragmentation production followed by $\chi_{c J} \rightarrow J / \psi+\gamma$ decays (non-prompt $J / \psi$ fragmentation production). In each channel, we took into account the contributions due to colour-singlet and colour-octet $c \bar{c}$ states. Apart from direct photoproduction, which had been considered to LO in Ref. [14], we also studied resolved photoproduction, both at NLO.

While the nonperturbative matrix elements multiplying the colour-singlet FF's may be reliably extracted from the decay properties of the charmonium states, the colour-octet matrix elements are adjusted to account for the enormous excess of recent Tevatron data on large- $p_{T}$ charmonium production over the theoretical prediction within the CSM. This procedure implicitly assumes, on the theoretical side, that there are no other production mechanisms that have been overlooked so far and, on the experimental side, that all backgrounds are fully under control. Therefore, the colour-octet matrix elements must be considered far less rigorously determined than the colour-singlet matrix elements. Obviously, it is of prime importance to probe the colour-octet matrix elements in other experiments as well. The signature for colour-octet $J / \psi$ production in $e^{+} e^{-}$annihilation 
at the $Z$-boson resonance has recently been investigated in Ref. [26, 34]. Here, we continued this research programme by assessing the prospects of the HERA experiments to substantiate the findings at the Tevatron.

Our main observations may be summarized as follows. As for direct $J / \psi$ photoproduction, fragmentation production starts to have a higher yield than the fusion mechanism of the CSM at $p_{T} \gtrsim 10 \mathrm{GeV}$ [see Figs. 1(a) and Z(a)]. This is in line with the main conclusion of the LO analysis of Ref. [14], since the NLO corrections are relatively modest in this case [see Fig. 3(a)]. However, in the range $p_{T} \lesssim 10 \mathrm{GeV}$, which will be accessed at HERA in the near future, resolved $J / \psi$ photoproduction via fragmentation, which has not yet been considered in the literature, is considerably more significant than direct photoproduction [see Figs. 1(b) and 2(b)], by a factor of 18 (2.3) at $p_{T}=3 \mathrm{GeV}(10 \mathrm{GeV})$. In fact, the resolved-photon fragmentation cross section already exceeds the fusion cross section at $p_{T} \gtrsim 5 \mathrm{GeV}$ and is comparable to it at smaller values of $p_{T}$. The bulk of this cross section originates in the subprocesses $g_{\gamma}+q_{p} \rightarrow g+X$ and $q_{\gamma}+g_{p} \rightarrow g+X$ followed by the fragmentation chain $g \rightarrow c \bar{c}\left[\underline{8},{ }^{3} S_{1}\right] \rightarrow J / \psi$. The dominance of resolved photoproduction is partly due to a large QCD $K$ factor, in excess of 2 [see Fig. 3(b)]. These results refer to the standard ranges $-1.5<y_{l a b}<1$ and $30 \mathrm{GeV}<W<150 \mathrm{GeV}$.

We suggested two ways to further enhance the (resolved-photon) fragmentation production relative to the fusion mechanism. One is to concentrate on the forward direction, e.g., $1<y_{l a b}<3.5$, where the resolved-photoproduction cross section peaks [see Fig. 2(b)]. The other one is to increase the CM energy available for the hard $\gamma p$ scattering, e.g., by choosing $150 \mathrm{GeV}<W<280 \mathrm{GeV}$, as the resolved-photoproduction cross section increases monotonically with $W$, while the fusion cross section takes its maximum at relatively low $W$ values (see Fig. (1). The usefulness of increasing $W$ and $y_{l a b}$ is nicely illustrated in Figs. 5 and 6, respectively. Both options should be feasible at HERA, and we look forward to the exciting scrutiny of the Tevatron $J / \psi$ colour-octet phenomenon by the HERA experiments in the near future.

\section{ACKNOWLEDGMENTS}

We are grateful to Reinhold Rückl for raising our interest in this study and for beneficial discussions at its early stages, to Eric Braaten for providing a computer code for the evaluation of $d_{g}\left[{ }^{1},{ }^{3} S_{1}\right](z)$ [5], to Eric Braaten and Sean Fleming for helpful technical advice concerning the numerical evolution of singular distributions in $z$ space, and to Kingman Cheung for a useful comment on the suppression of the colour-octet contribution to the $c \rightarrow J / \psi$ FF. One of us (G.K.) is grateful to the Theory Group of the Werner-HeisenbergInstitut for the hospitality extended to him during a visit when this paper was prepared.

\section{A Appendix: Coefficients of the perturbative frag- mentation functions}

For the reader's convenience, we collect here the colour-singlet and colour-octet coefficient functions, at the starting scale $\mu_{0}$, for the gluon and charm-quark FF's into $J / \psi$ and $\chi_{c J}$ 
mesons, with $J=0,1,2$. The $g \rightarrow c \bar{c}\left[\underline{1},{ }^{3} S_{1}\right]$ coefficient function has the two-dimensional integral representation [5]

$$
\begin{aligned}
& \left.d_{g}^{[1},{ }^{3} S_{1}\right](z)=\frac{5}{5184 \pi} \int_{0}^{z} d r \int_{\left(r+z^{2}\right) /(2 z)}^{(1+r) / 2} d y \frac{1}{(1-y)^{2}(y-r)^{2}\left(y^{2}-r\right)^{2}} \\
& \times \sum_{i=0}^{2} z^{i}\left(f_{i}(r, y)+g_{i}(r, y) \frac{1+r-2 y}{2(y-r) \sqrt{y^{2}-r}} \ln \frac{y-r+\sqrt{y^{2}-r}}{y-r-\sqrt{y^{2}-r}}\right) \text {, }
\end{aligned}
$$

where

$$
\begin{aligned}
f_{0}(r, y)= & r^{2}(1+r)\left(3+12 r+13 r^{2}\right)-16 r^{2}(1+r)(1+3 r) y \\
& -2 r\left(3-9 r-21 r^{2}+7 r^{3}\right) y^{2}+8 r\left(4+3 r+3 r^{2}\right) y^{3}-4 r\left(9-3 r-4 r^{2}\right) y^{4} \\
& -16\left(1+3 r+3 r^{2}\right) y^{5}+8(6+7 r) y^{6}-32 y^{7}, \\
f_{1}(r, y)= & -2 r\left(1+5 r+19 r^{2}+7 r^{3}\right) y+96 r^{2}(1+r) y^{2}+8\left(1-5 r-22 r^{2}-2 r^{3}\right) y^{3} \\
& +16 r(7+3 r) y^{4}-8(5+7 r) y^{5}+32 y^{6}, \\
f_{2}(r, y)= & \left(1+5 r+19 r^{2}+7 r^{3}\right)-48 r^{2}(1+r) y-4\left(1-5 r-22 r^{2}-2 r^{3}\right) y^{2} \\
& -8 r(7+3 r) y^{3}+4(5+7 r) y^{4}-16 y^{5}, \\
g_{0}(r, y)= & r^{3}(1-r)\left(3+24 r+13 r^{2}\right)-4 r^{3}\left(7-3 r-12 r^{2}\right) y-2 r^{3}\left(17+22 r-7 r^{2}\right) y^{2} \\
& +4 r^{2}\left(13+5 r-6 r^{2}\right) y^{3}-8 r\left(1+2 r+5 r^{2}+2 r^{3}\right) y^{4}-8 r\left(3-11 r-6 r^{2}\right) y^{5} \\
& +8\left(1-2 r-5 r^{2}\right) y^{6}, \\
g_{1}(r, y)= & -2 r^{2}(1+r)(1-r)(1+7 r) y+8 r^{2}(1+3 r)(1-4 r) y^{2} \\
& +4 r\left(1+10 r+57 r^{2}+4 r^{3}\right) y^{3}-8 r\left(1+29 r+6 r^{2}\right) y^{4}-8\left(1-8 r-5 r^{2}\right) y^{5}, \\
g_{2}(r, y)= & r^{2}(1+r)(1-r)(1+7 r)-4 r^{2}(1+3 r)(1-4 r) y \\
& -2 r\left(1+10 r+57 r^{2}+4 r^{3}\right) y^{2}+4 r\left(1+29 r+6 r^{2}\right) y^{3}+4\left(1-8 r-5 r^{2}\right) y^{4} .
\end{aligned}
$$

All other coefficient functions are available in closed form [0, 19, 20,21]:

$$
\begin{aligned}
& d_{g}^{\left[\underline{8},{ }^{3} S_{1}\right]}(z)=\frac{\pi}{24} \delta(1-z), \\
& d_{g}^{\left[\underline{1},{ }^{3} P_{0}\right]}(z)=\frac{2}{81}\left[\frac{1}{4} \delta(1-z)+\left(\frac{1}{1-z}\right)_{+}-1+\frac{85}{8} z-\frac{13}{4} z^{2}+\frac{9}{4}(5-3 z) \ln (1-z)\right], \\
& d_{g}^{\left[\underline{1},{ }^{3} P_{1}\right]}(z)=\frac{2}{81}\left[\frac{1}{8} \delta(1-z)+\left(\frac{1}{1-z}\right)_{+}-1-\frac{1}{4} z-z^{2}\right], \\
& d_{g}^{\left[\underline{1},{ }^{3} P_{2}\right]}(z)=\frac{2}{81}\left[\frac{7}{40} \delta(1-z)+\left(\frac{1}{1-z}\right)_{+}-1+\frac{11}{4} z-z^{2}+\frac{9}{5}(2-z) \ln (1-z)\right], \\
& \left.d_{c}^{\left[\underline{1},{ }^{3} S_{1}\right]}(z)=\frac{32}{3} d_{c}^{[}{ }^{3}{ }^{3} S_{1}\right](z)=\frac{16 z(1-z)^{2}}{243(2-z)^{6}}\left(16-32 z+72 z^{2}-32 z^{3}+5 z^{4}\right), \\
& d_{c}^{\left[\underline{1},{ }^{3} P_{0}\right]}(z)=\frac{16 z(1-z)^{2}}{729(2-z)^{8}}\left(192+384 z+528 z^{2}-1376 z^{3}+1060 z^{4}-376 z^{5}+59 z^{6}\right), \\
& d_{c}^{\left[\underline{1},{ }^{3} P_{1}\right]}(z)=\frac{64 z(1-z)^{2}}{729(2-z)^{8}}\left(96-288 z+496 z^{2}-408 z^{3}+202 z^{4}-54 z^{5}+7 z^{6}\right),
\end{aligned}
$$




$$
d_{c}^{\left[1,{ }^{3} P_{2}\right]}(z)=\frac{128 z(1-z)^{2}}{3645(2-z)^{8}}\left(48-192 z+480 z^{2}-668 z^{3}+541 z^{4}-184 z^{5}+23 z^{6}\right) .
$$

As usual, the plus distribution $1 /(1-z)_{+}$is defined by $\int_{0}^{1} d z f(z) /(1-z)_{+}=\int_{0}^{1} d z[f(z)-$ $f(1)] /(1-z)$ for any regular function $f(z)$.

For the numerical solution of the $\mu^{2}$-evolution equations in $z$ space, it is useful to approximate

$$
\begin{aligned}
& \delta(1-z)= \begin{cases}0, & \text { if } z \leq 1-\epsilon, \\
\frac{1}{\epsilon}, & \text { if } 1-\epsilon<z \leq 1,\end{cases} \\
& \left(\frac{1}{1-z}\right)_{+}= \begin{cases}\frac{1}{1-z}, & \text { if } z \leq 1-\epsilon, \\
\frac{\ln \epsilon}{\epsilon}, & \text { if } 1-\epsilon<z \leq 1,\end{cases} \\
& \ln (1-z)= \begin{cases}\ln (1-z), & \text { if } z \leq 1-\epsilon, \\
\ln \epsilon-1, & \text { if } 1-\epsilon<z \leq 1,\end{cases}
\end{aligned}
$$

with appropriate $0<\epsilon \ll 1$. We have verified, for the perturbative FF's of Ref. [27] in $\mathrm{LO}$ and NLO, that the $\mu^{2}$ evolution in $z$ space implemented using Eq. (22) agrees very well with the $\mu^{2}$ evolution in Mellin space [35.

\section{References}

[1] E.L. Berger and D. Jones, Phys. Rev. D 23, 1521 (1981); R. Baier and R. Rückl, Phys. Lett. 102B, 364 (1981); Nucl. Phys. B208, 381 (1982); ibid. B218, 289 (1983); J.G. Körner, J. Cleymans, M. Kuroda, and G.J. Gounaris, ibid. B204, 6 (1982); for a review, see G.A. Schuler, CERN Report Nr. CERN-TH.7170/94 (unpublished).

[2] R. Baier and R. Rückl, Z. Phys. C 19, 251 (1983).

[3] E.W.N. Glover, A.D. Martin, and W.J. Stirling, Z. Phys. C 38, 473 (1988).

[4] CDF Collaboration, F. Abe et al., Phys. Rev. Lett. 69, 3704 (1992); ibid. 71, 2537 (1993).

[5] E. Braaten and T.C. Yuan, Phys. Rev. Lett. 71, 1673 (1993); Phys. Rev. D 52, 6627 (1995); see also the subsequent papers in Refs. [25.30].

[6] H1 Collaboration, S. Aid et al., Nucl. Phys. B472, 3 (1996).

[7] M. Krämer, J. Zunft, J. Steegborn, and P.M. Zerwas, Phys. Lett. B 348, 657 (1995); M. Krämer, Nucl. Phys. B459, 3 (1996). 
[8] H. Jung, G.A. Schuler, and J. Terrón, in Proceedings of the Workshop on Physics at HERA, Hamburg, Germany, 29-30 October 1991, edited by W. Buchmüller and G. Ingelman, Vol. 2, p. 712; Int. J. Mod. Phys. A 7, 7955 (1992).

[9] P. Cho and A.K. Leibovich, Phys. Rev. D 53, 150 (1996); ibid. 53, 6203 (1996).

[10] M. Cacciari and M. Krämer, Phys. Rev. Lett. 76, 4128 (1996); P. Ko, J. Lee, and H.S. Song, Phys. Rev. D 54, 4312 (1996).

[11] M. Cacciari and M. Krämer, in Proceedings of the Workshop 1995/96 on Future Physics at HERA, edited by G. Ingelman, A. De Roeck, and R. Klanner, Vol. 1, p. 416.

[12] E. Braaten and S. Fleming, Phys. Rev. Lett. 74, 3327 (1995).

[13] M. Cacciari, M. Greco, M.L. Mangano, and A. Petrelli, Phys. Lett. B 356, 553 (1995).

[14] R.M. Godbole, D.P. Roy, and K. Sridhar, Phys. Lett. B 373, 328 (1996); R.M. Godbole (private communication).

[15] V.A. Saleev, Mod. Phys. Lett. A 9, 1083 (1994).

[16] B.A. Kniehl, G. Kramer, and M. Spira, Report Nos. CERN-TH/96-274, DESY 96210, MPI/PhT/96-103, and hep-ph/9610267 (October 1996), Z. Phys. C (in press).

[17] P. Aurenche, R. Baier, A. Douiri, M. Fontannaz, and D. Schiff, Nucl. Phys. B286, 553 (1987); F. Aversa, P. Chiappetta, M. Greco, and J.Ph. Guillet, Nucl. Phys. B327, 105 (1989).

[18] G.T. Bodwin, E. Braaten, and G.P. Lepage, Phys. Rev. D 51, 1125 (1995); 55, 5853(E) (1997).

[19] E. Braaten and T.C. Yuan, Phys. Rev. D 50, 3176 (1994); E. Braaten and Y.-Q. Chen, Phys. Rev. D 55, 2693 (1997).

[20] E. Braaten, K. Cheung, and T.C. Yuan, Phys. Rev. D 48, 4230 (1993); Y.-Q. Chen, Phys. Rev. D 48, 5181 (1993); 50, 6013(E) (1994).

[21] T.C. Yuan, Phys. Rev. D 50, 5664 (1994).

[22] E. Braaten, S. Fleming, and T.C. Yuan, Annu. Rev. Nucl. Part. Sci. 46, 197 (1996).

[23] Particle Data Group, R.M. Barnett et al., Phys. Rev. D 54, 1 (1996).

[24] G. Curci, W. Furmanski, and R. Petronzio, Nucl. Phys. B175, 27 (1980); W. Furmanski and R. Petronzio, Phys. Lett. 97B, 437 (1980); P.J. Rijken and W.L. van Neeren, Nucl. Phys. B487, 233 (1997); M. Stratmann and W. Vogelsang, Report Nos. DO-TH 96/23, RAL-TR-96-097, and hep-ph/9612250 (December 1996). 
[25] E. Braaten, M.A. Doncheski, S. Fleming, and M.L. Mangano, Phys. Lett. B 333, 548 (1994).

[26] P. Ernström, L. Lönnblad, and M. Vänttinen, NORDITA Report Nos. NORDITA96/78 P and hep-ph/9612408 (December 1996).

[27] B. Mele and P. Nason, Nucl. Phys. B361, 626 (1991); see also P. Nason, S. Dawson, and R.K. Ellis, Nucl. Phys. B327, 49 (1989); B335, 260(E) (1990).

[28] H.L. Lai, J. Huston, S. Kuhlmann, F. Olness, J. Owens, D. Soper, W.K. Tung, and H. Weerts, Phys. Rev. D 55, 1280 (1997).

[29] M. Glück, E. Reya, and A. Vogt, Phys. Rev. D 46, 1973 (1992).

[30] M. Cacciari and M. Greco, Phys. Rev. Lett. 73, 1586 (1994); D.P. Roy and K. Sridhar, Phys. Lett. B 339, 141 (1994).

[31] H1 Collaboration, S. Aid et al., Nucl. Phys. B472, 32 (1996); ZEUS Collaboration, Contributed Paper No. pa05-051 to the 28th International Conference on High Energy Physics, Warsaw, Poland, 25-31 July 1996.

[32] R. Brugnera, C. Coldewey, C. Kiesling, B. Naroska, and A. Wegner, private communications.

[33] B.A. Kniehl and G. Kramer, Report Nos. DESY 96-036, MPI/PhT/96-018, and hep-ph/9703280 (March 1997).

E. Braaten and Y.-Q. Chen, Phys. Rev. Lett. 76, 730 (1996);

[34] E. Braaten and Y.-Q. Chen, Phys. Rev. Lett. 76, 730 (1996); K. Cheung, W.-Y. Keung, and T.C. Yuan, Phys. Rev. Lett. 76, 877 (1996); F. Yuan, C.-F. Qiao, and K.T. Chao, Peking University Report Nos. PUTP-96-31 and hep-ph/9701361 (January 1997).

[35] M. Cacciari, M. Greco, B.A. Kniehl, M. Krämer, G. Kramer, and M. Spira, Nucl. Phys. B466, 173 (1996). 


\section{FIGURE CAPTIONS}

Figure 1: (a) Direct-photon, (b) resolved-photon, and (c) total contributions to the cross section $d \sigma / d p_{T}$ of inclusive $J / \psi$ photoproduction via fragmentation at HERA, integrated over $30 \mathrm{GeV}<W<150 \mathrm{GeV}$ and $-1.5<y_{l a b}<1$. The NLO contributions due to prompt singlet (P1), prompt octet (P8), non-prompt singlet plus octet fragmentation $(\mathrm{N} 1+8)$, and their sum (frag.) are compared with the LO CSM contribution (fusion).

Figure 2: (a) Direct-photon, (b) resolved-photon, and (c) total contributions to the cross section $d \sigma / d y_{\text {lab }} p_{T}$ of inclusive $J / \psi$ photoproduction via fragmentation at HERA, integrated over $30 \mathrm{GeV}<W<150 \mathrm{GeV}$, at $p_{T}=5$ and $10 \mathrm{GeV}$. The NLO contributions due to prompt singlet (P1), prompt octet (P8), non-prompt singlet plus octet fragmentation $(\mathrm{N} 1+8)$, and their sum (frag.) are compared with the LO CSM contribution (fusion).

Figure 3: NLO to LO ratios for the (a) direct-photon, (b) resolved-photon, and (c) total contributions to the cross section $d \sigma / d p_{T}$ of inclusive $J / \psi$ photoproduction via fragmentation at HERA, integrated over $30 \mathrm{GeV}<W<150 \mathrm{GeV}$ and $-1.5<y_{\text {lab }}<1$. The ratios are taken for prompt singlet (P1), prompt octet (P8), non-prompt singlet plus octet fragmentation $(\mathrm{N} 1+8)$, and their sum (frag.).

Figure 4: Cross section $d^{2} \sigma / d W d p_{T}$ of inclusive $J / \psi$ photoproduction via fragmentation at HERA, integrated over $-1.5<y_{\text {lab }}<1$, at $p_{T}=5$ and $10 \mathrm{GeV}$. The NLO fragmentation contributions due direct photons, resolved photons, and their sum are compared with the LO CSM contribution.

Figure 5: NLO fragmentation and LO CSM contributions to the cross section $d \sigma / d p_{T}$ of inclusive $J / \psi$ photoproduction at HERA, integrated over $-1.5<y_{\text {lab }}<1$ and two different $W$ intervals, namely, $30 \mathrm{GeV}<W<150 \mathrm{GeV}$ and $150 \mathrm{GeV}<W<280 \mathrm{GeV}$.

Figure 6: NLO fragmentation and LO CSM contributions to the cross section $d \sigma / d p_{T}$ of inclusive $J / \psi$ photoproduction at HERA, integrated over $150 \mathrm{GeV}<W<280 \mathrm{GeV}$ and two different $y_{l a b}$ intervals, namely, $-1.5<y_{l a b}<1$ and $1<y_{l a b}<3.5$. 


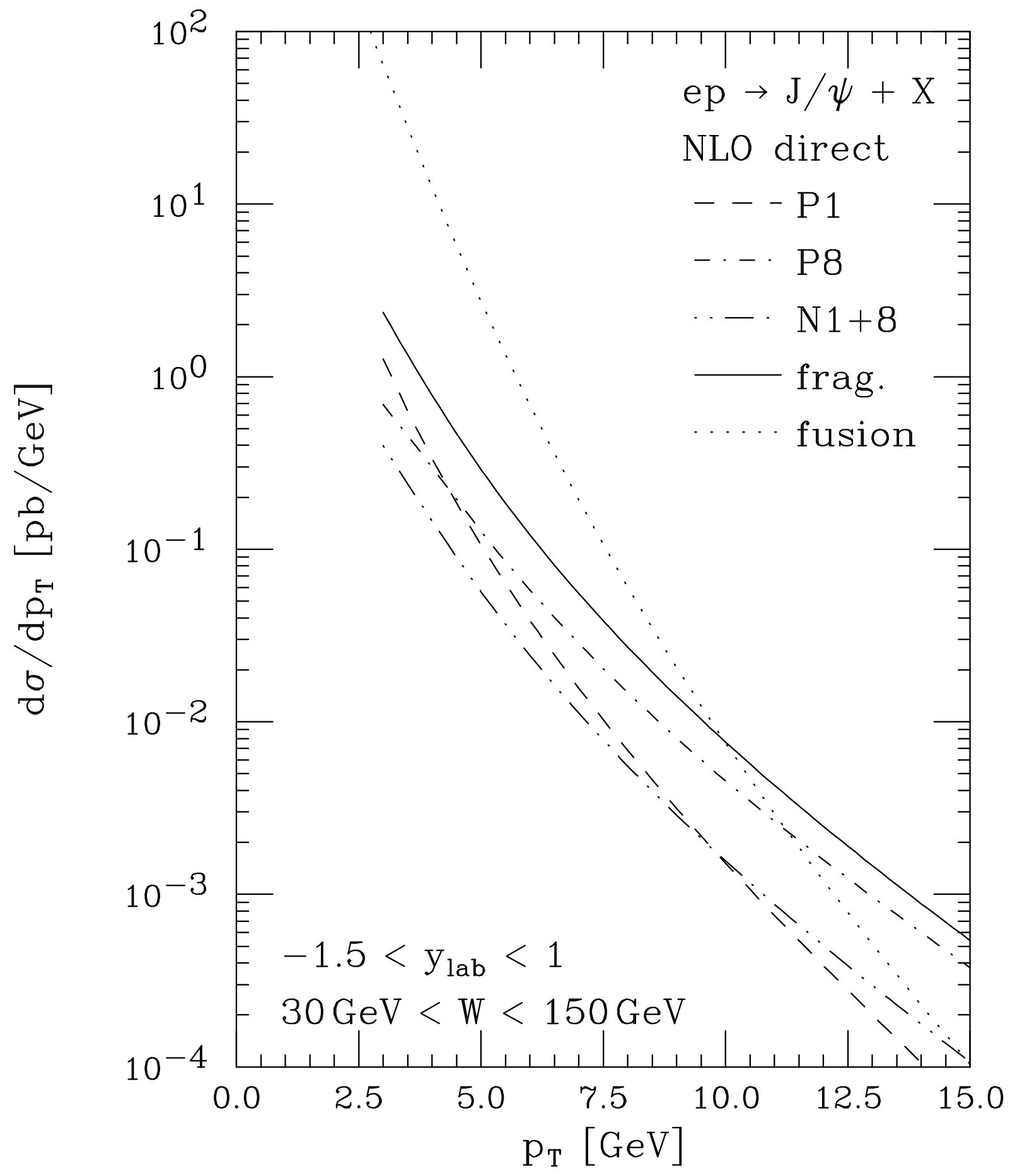

Fig. 1a 


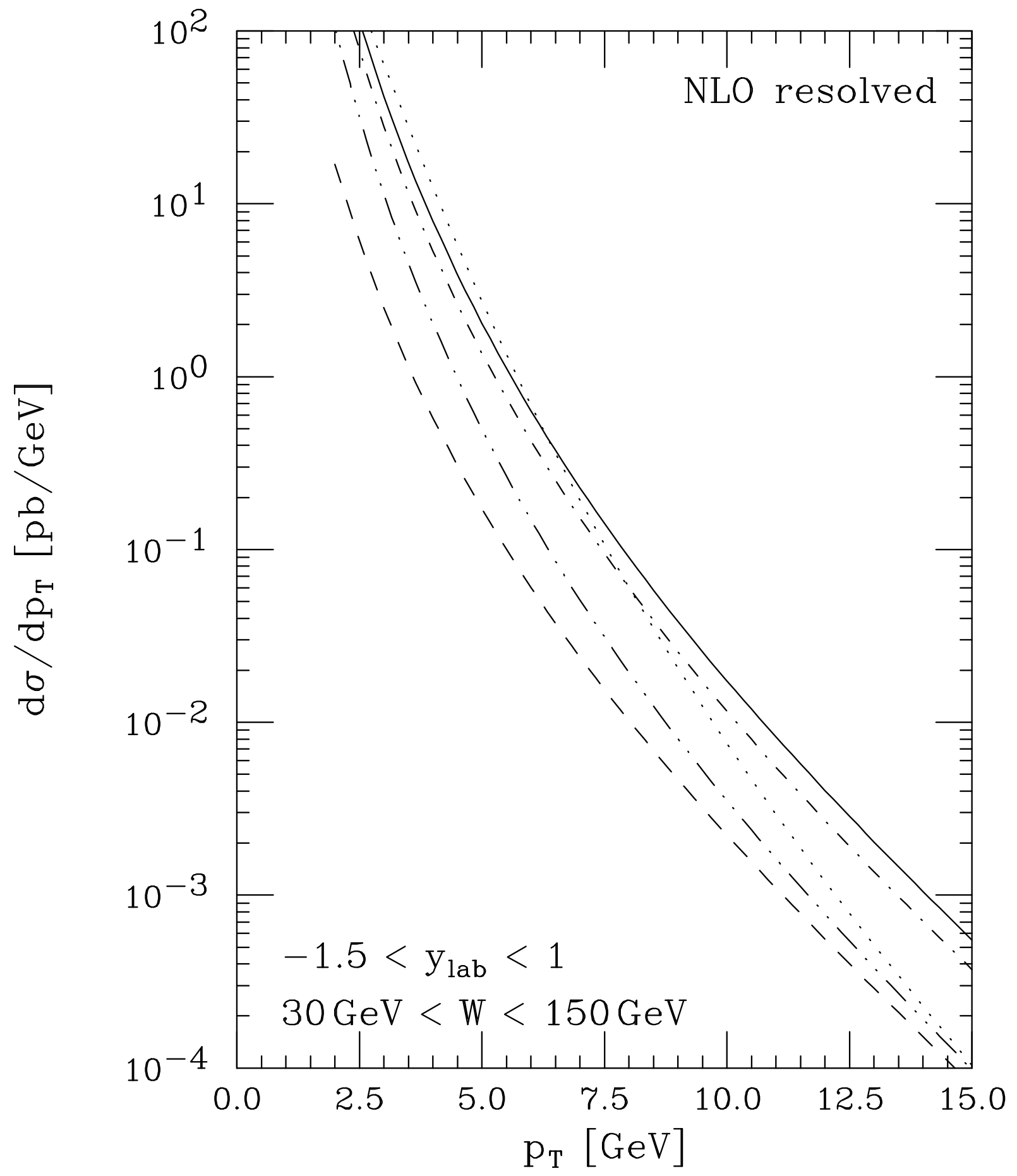

Fig. 1b 


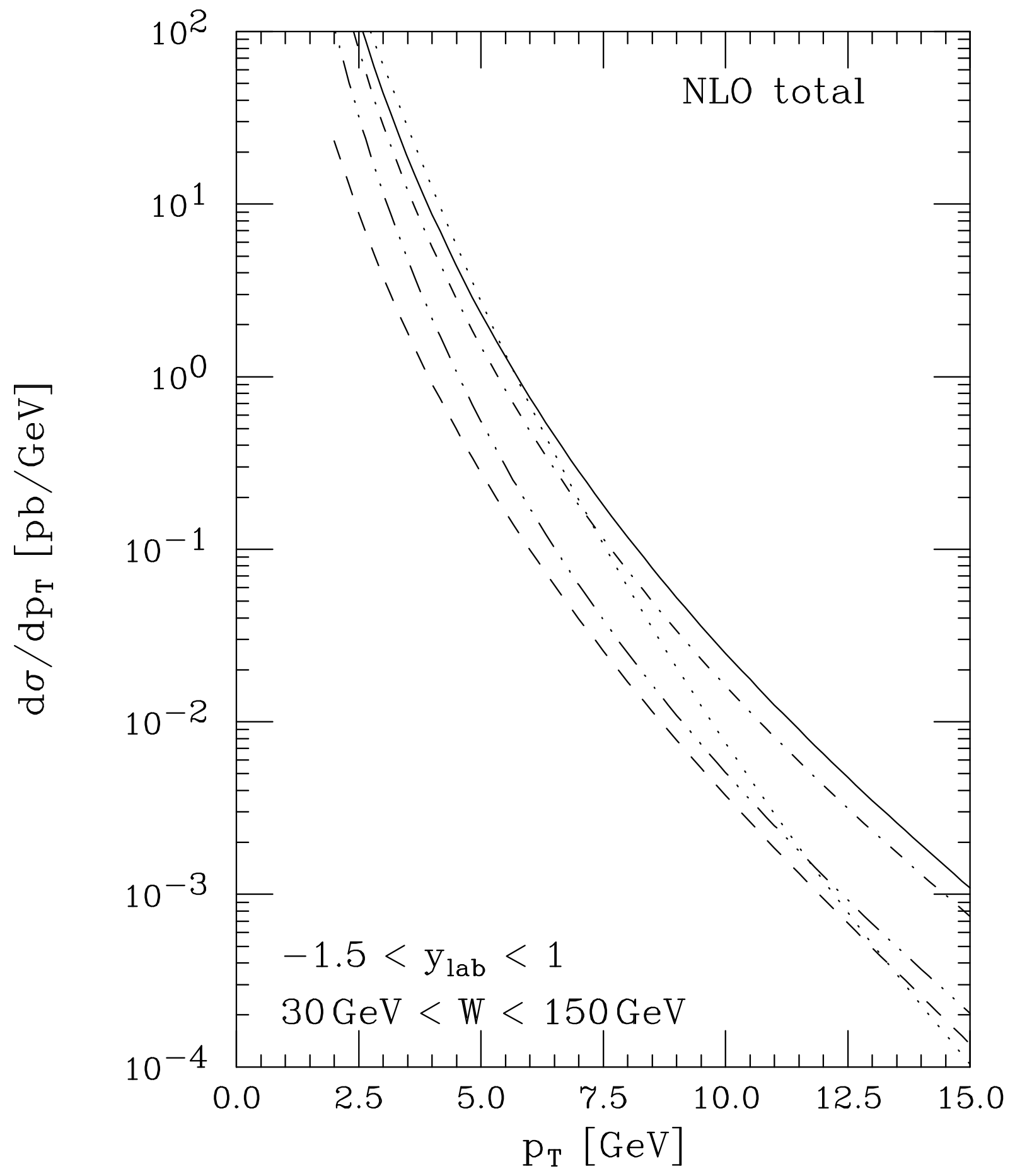

Fig. 1c 


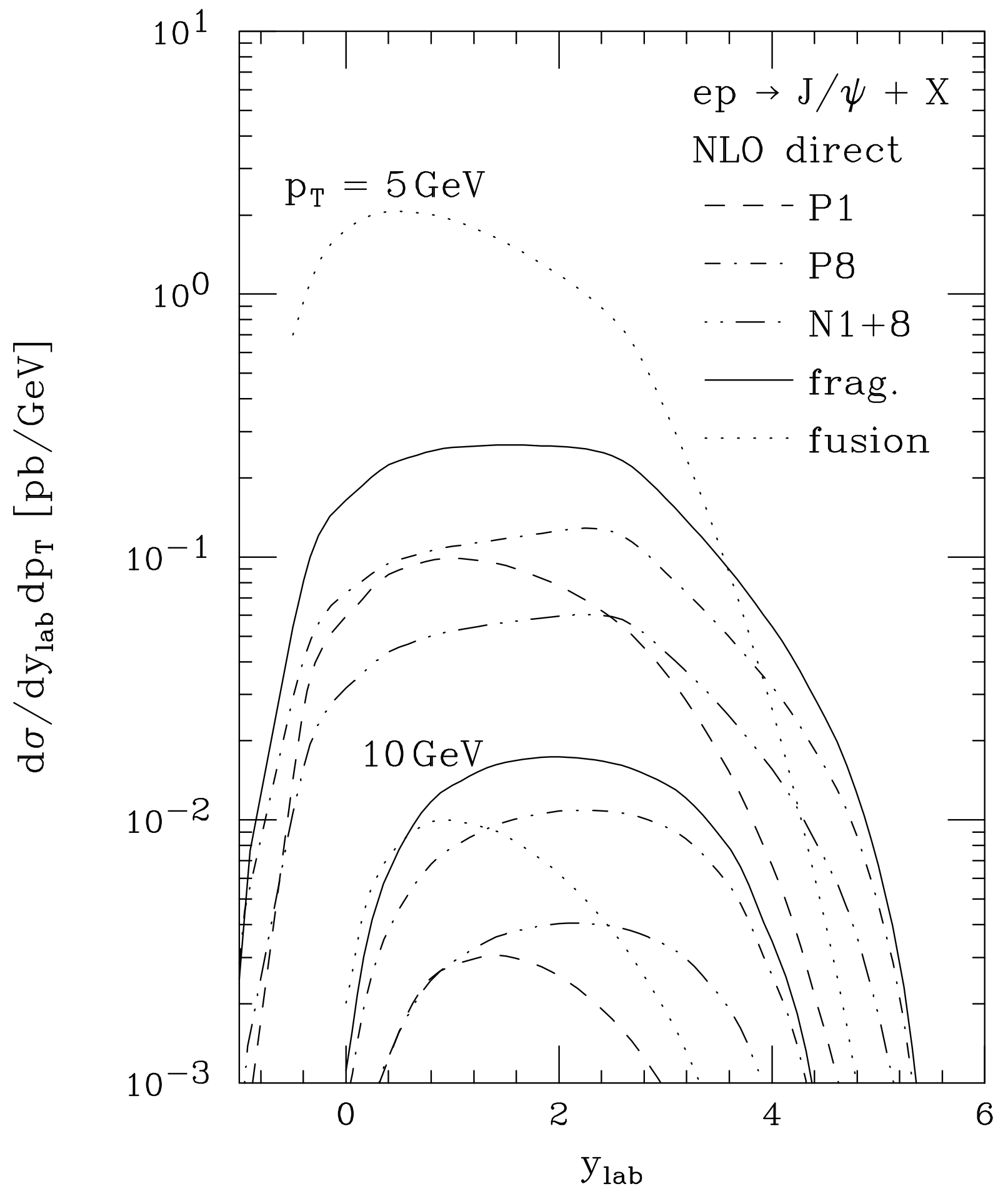

Fig. 2a 


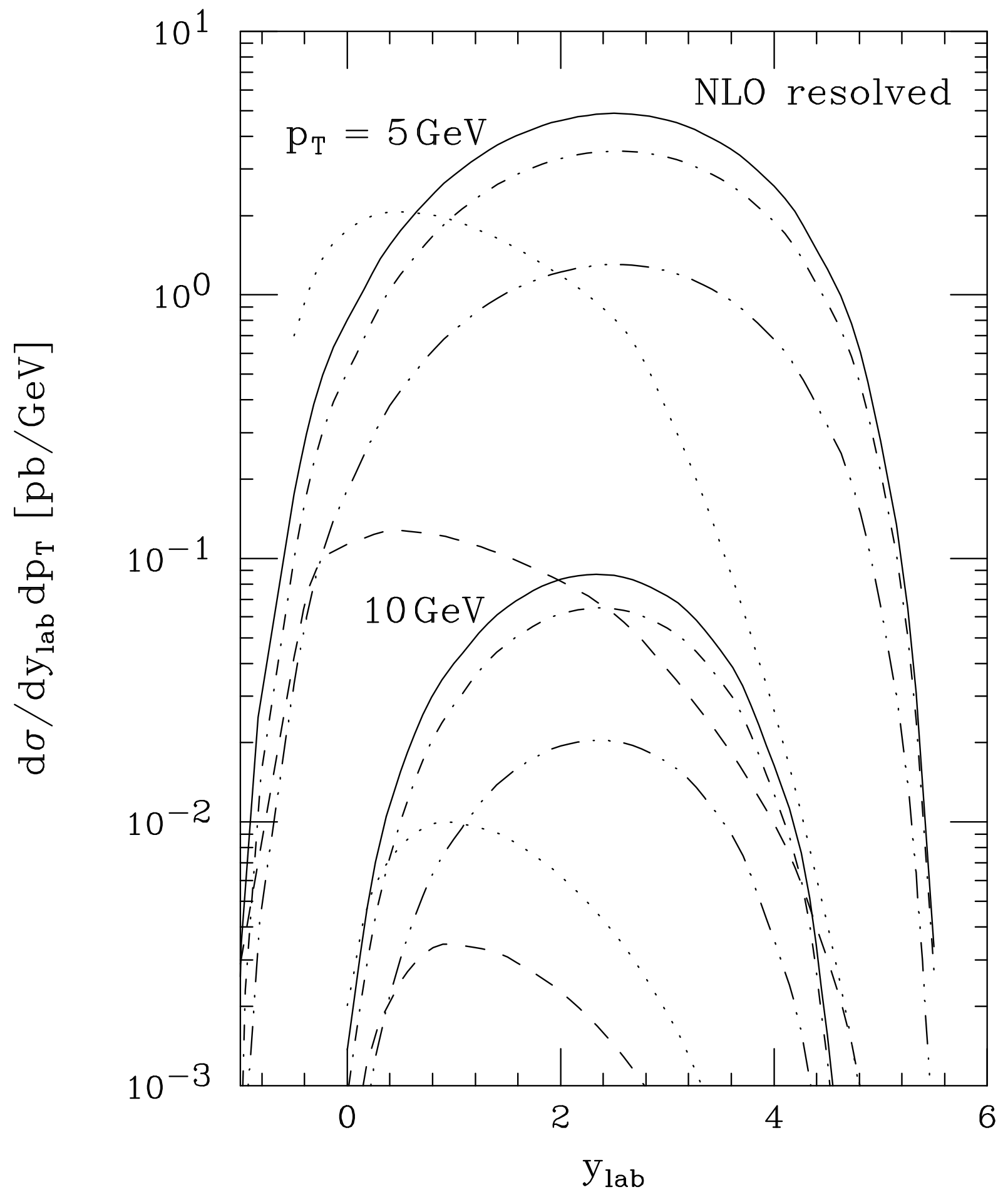

Fig. 2b 


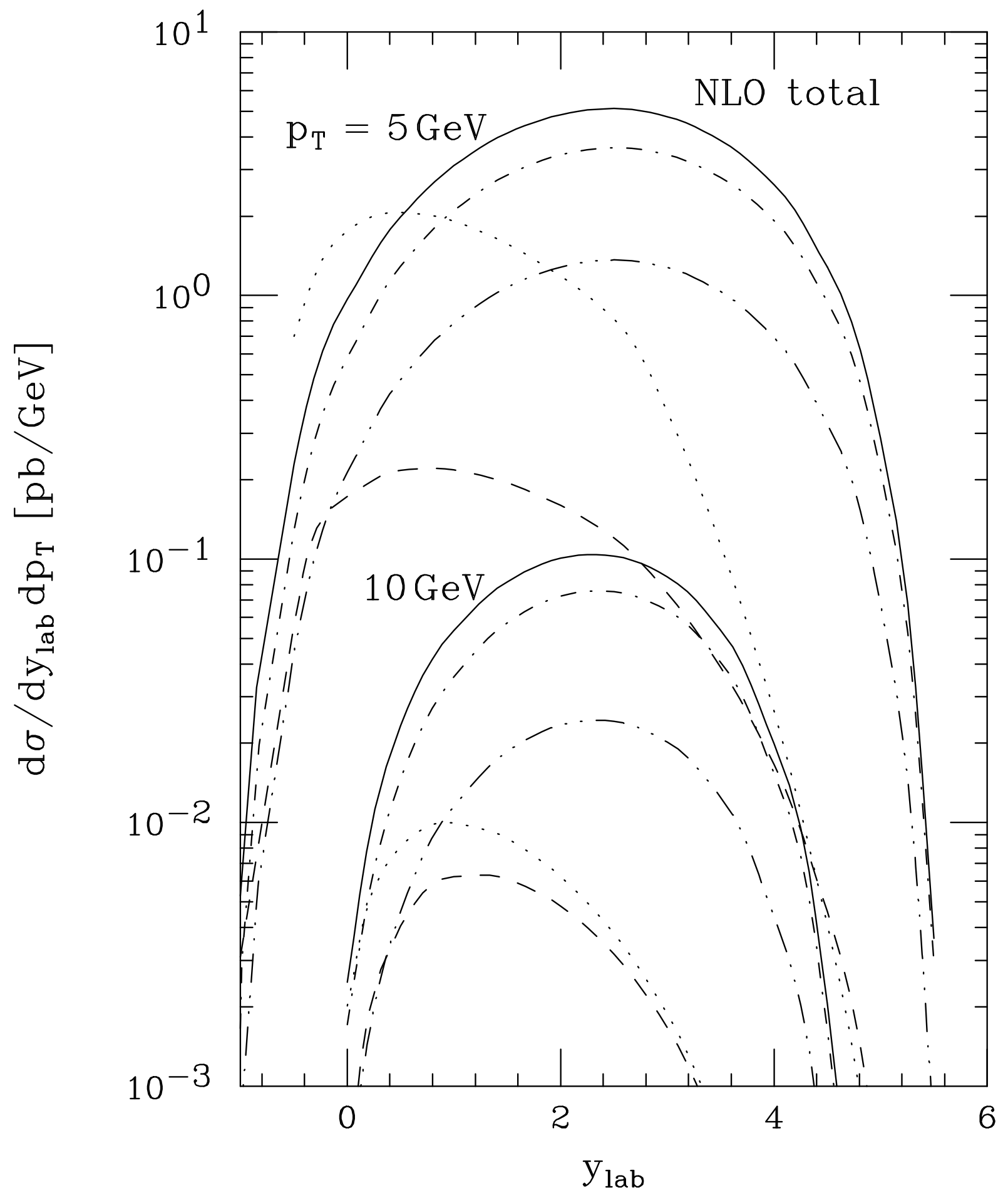

Fig. 2c 
0
0
0
0
0
0
0
0
0
0
$\frac{1}{E}$
0
0
0
0
0

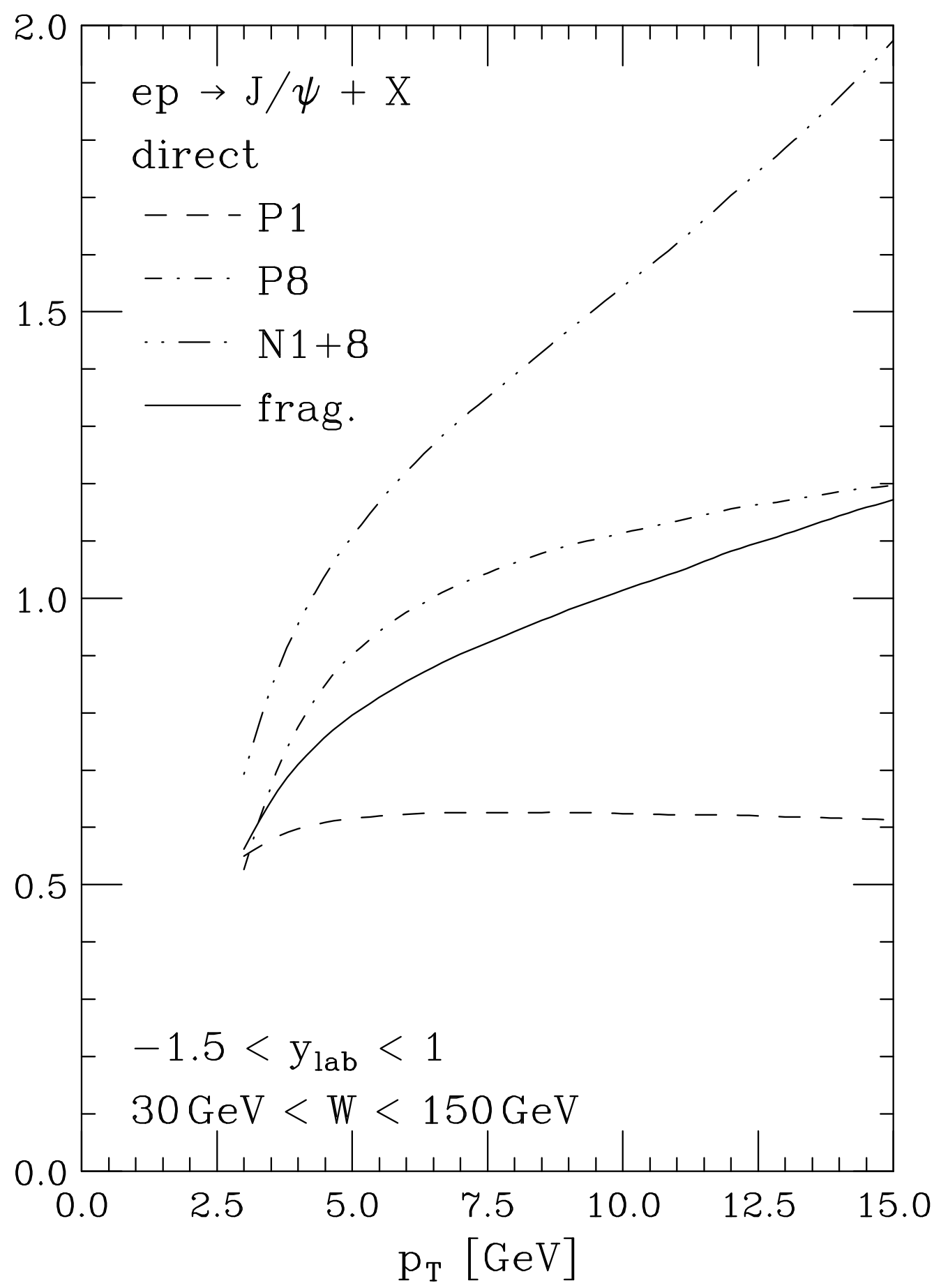

Fig. 3a 


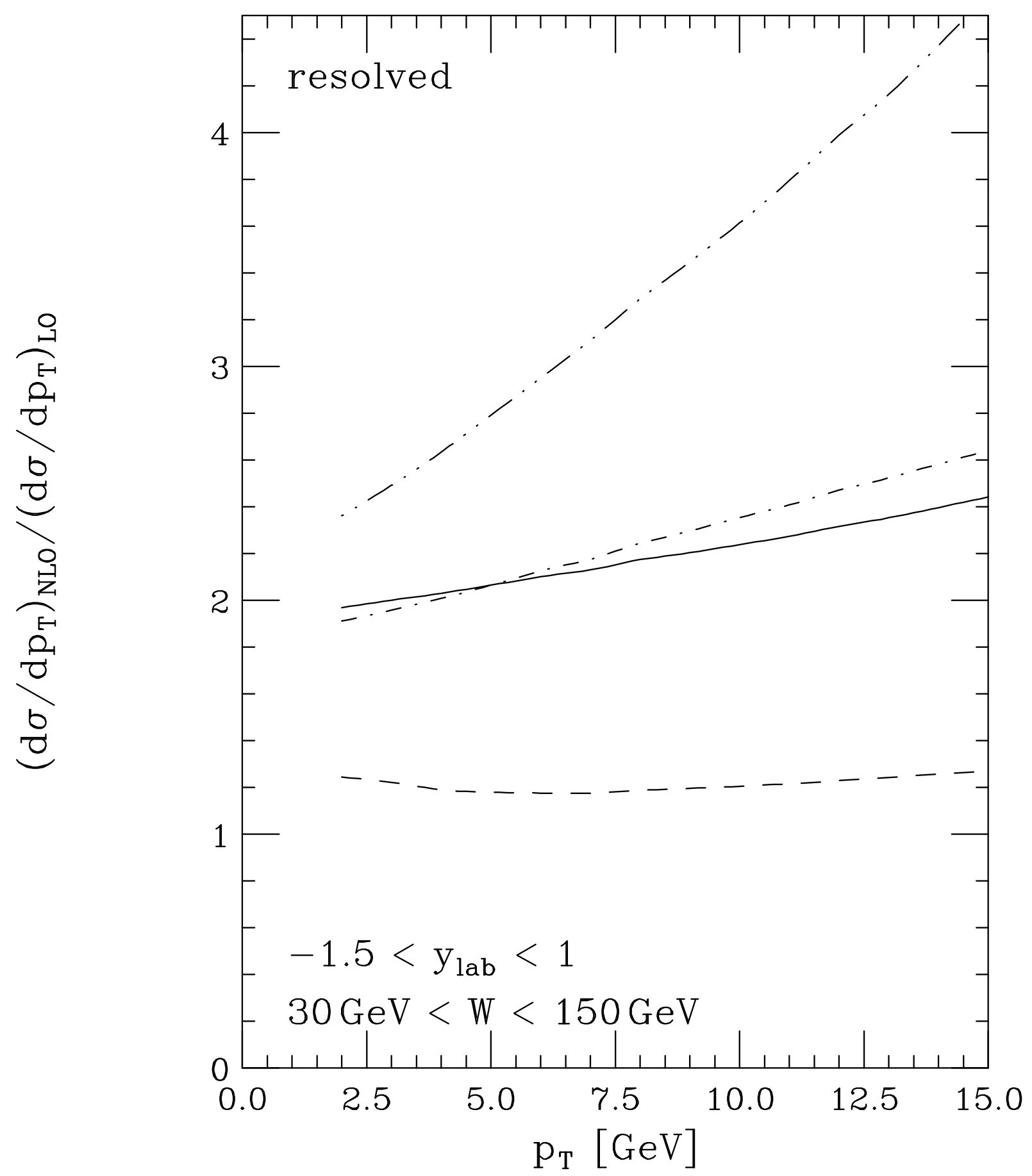

Fig. 3b 


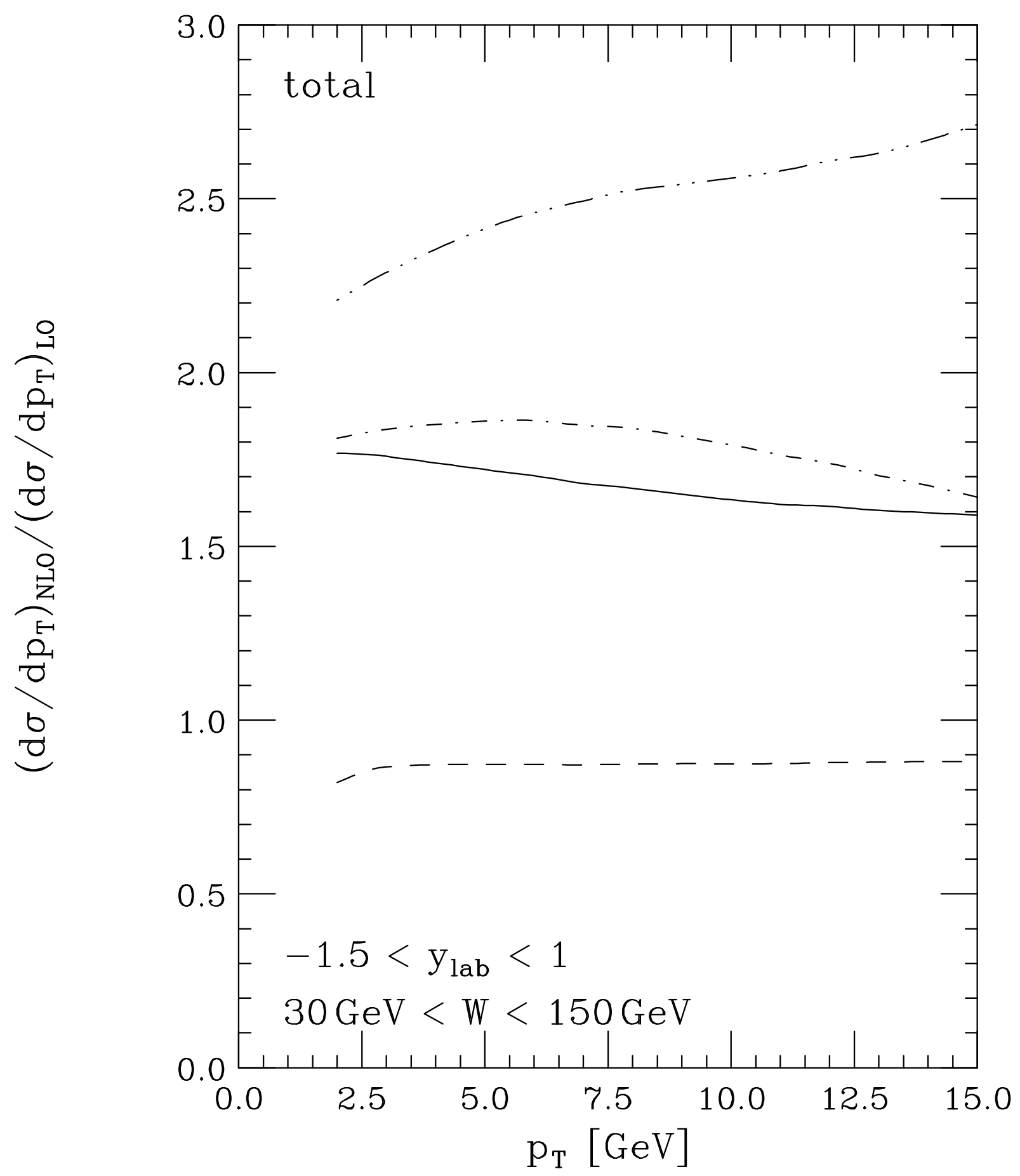

Fig. 3c 


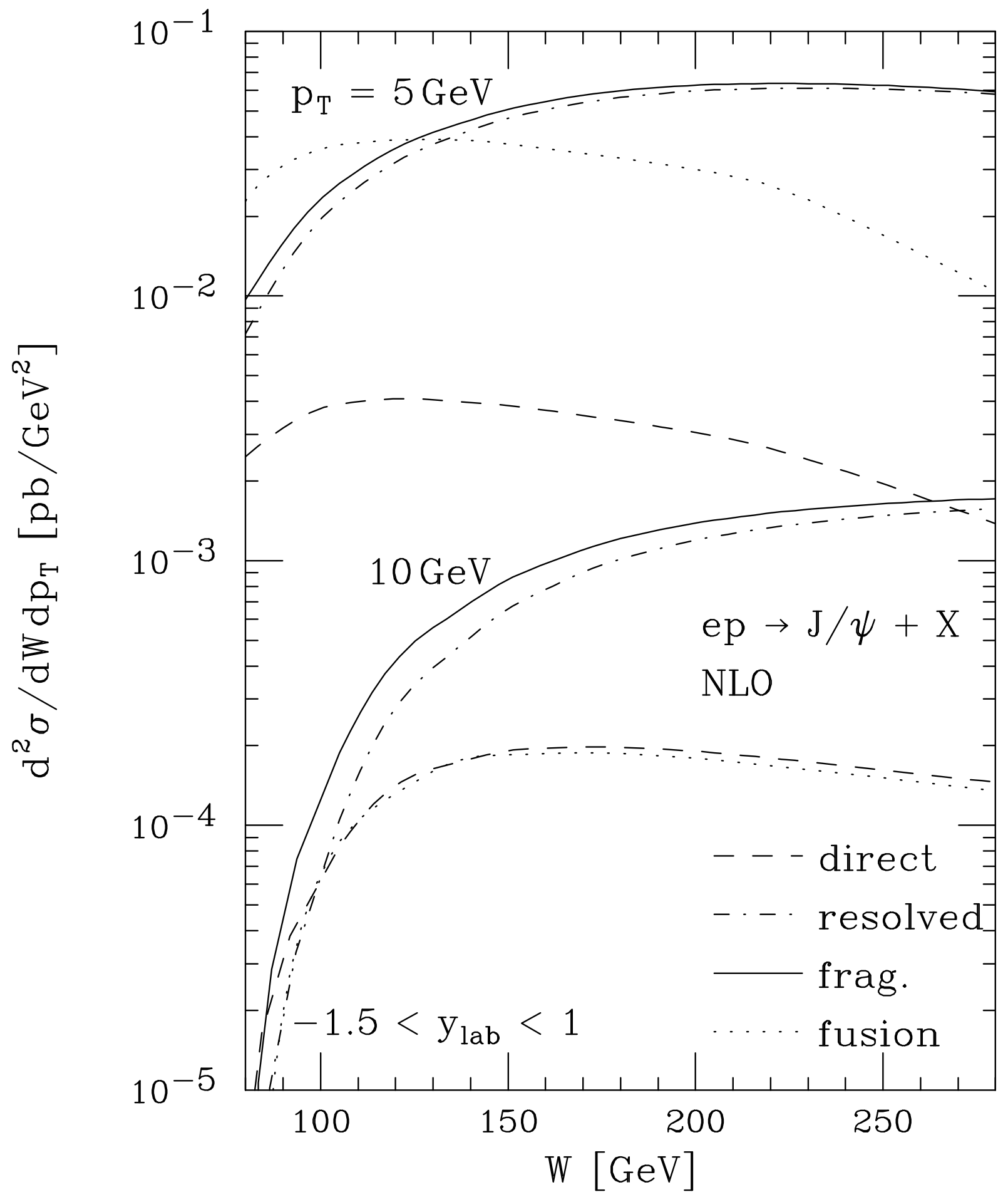

Fig. 4 


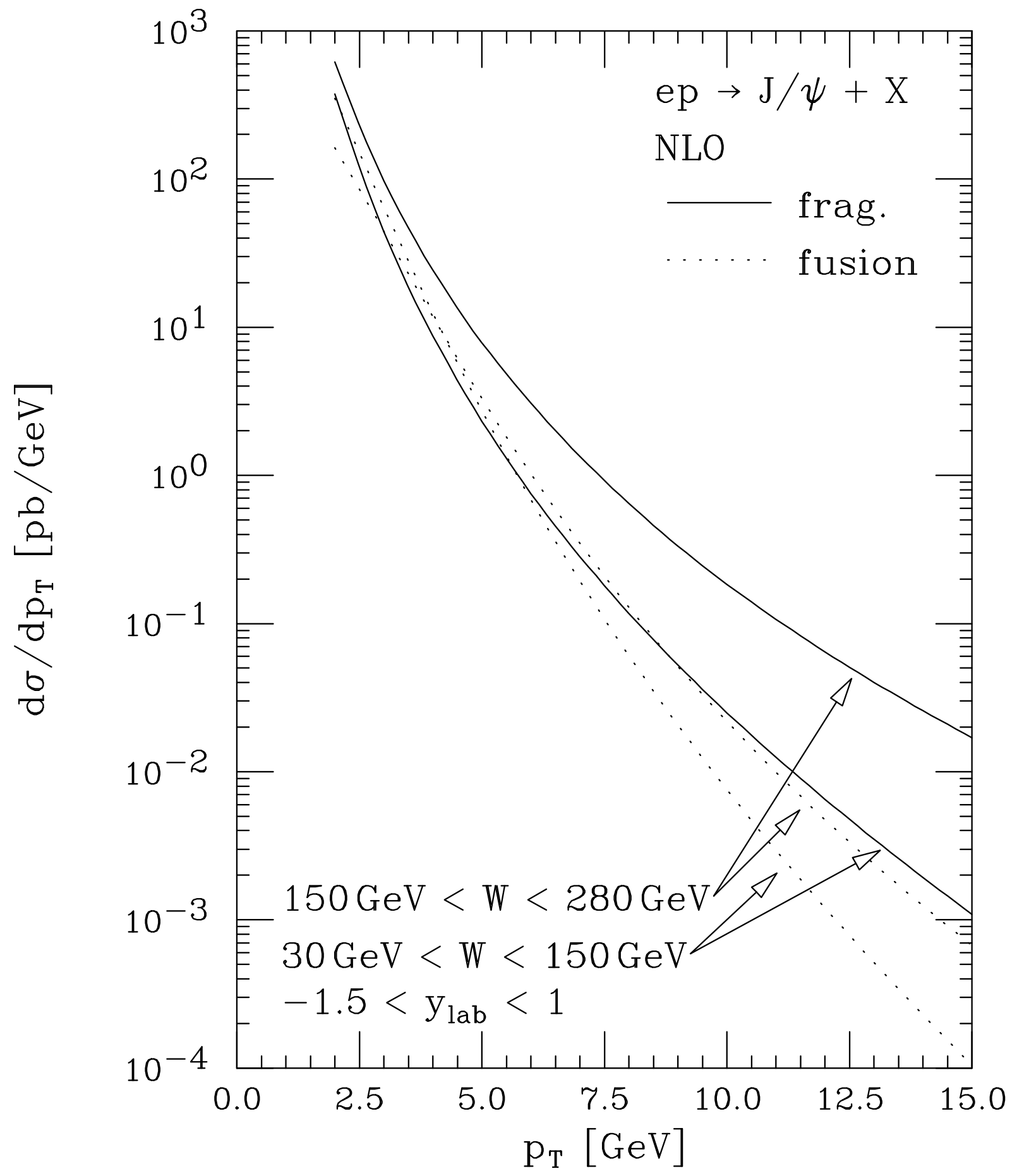

Fig. 5 


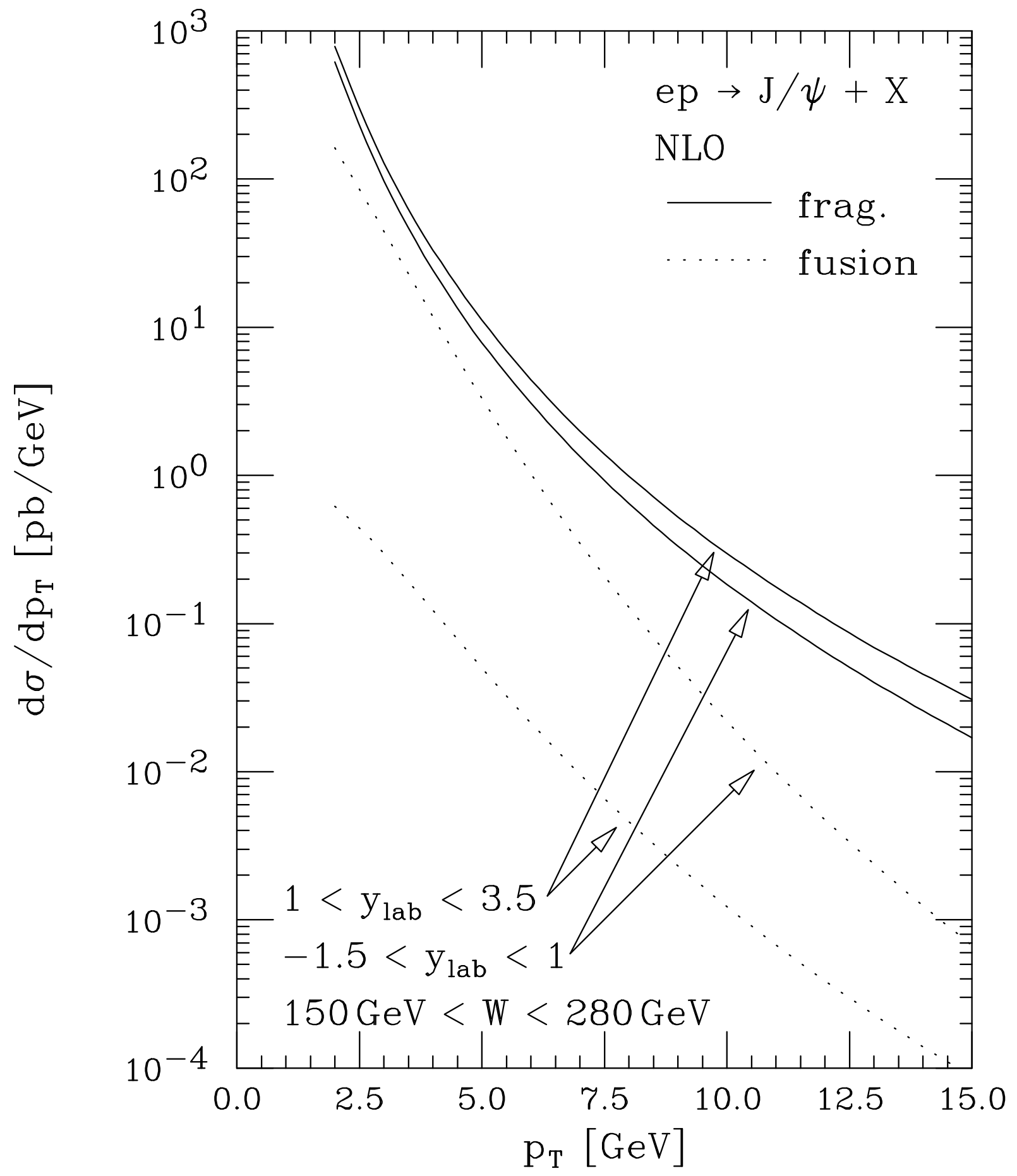

Fig. 6 